\title{
EXISTENCE OF TRAVELLING WAVE SOLUTIONS FOR REACTION-DIFFUSION-CONVECTION SYSTEMS VIA THE CONLEY INDEX THEORY
}

\author{
BOGDAN KAŹMIERCZAK
}

\begin{abstract}
By using the Conley connection index theory we prove the existence of travelling wave solutions for a class of reaction-diffusion systems.

The results are applied to equations describing laser sustained plasma.
\end{abstract}

\section{Introduction}

Consider a system of reaction-diffusion-convection equations

(1) $\widetilde{c}_{i}(u, \nabla u)\left(\frac{\partial}{\partial t}+\vec{v}_{i}(u, \nabla u) \cdot \nabla\right) u_{i}=\widetilde{a}\left(u, \nabla u_{i}\right) \Delta u_{i}+\widetilde{M}_{i}(u, \nabla u) \cdot \nabla u_{i}+f_{i}(u)$, where $(t, x) \in(-\infty, \infty) \times \mathbb{R}^{d}, d \geq 1, i=1, \ldots, n, n \geq 2, u=\left(u_{1}, \ldots, u_{n}\right)$.

Let us look for travelling wave type solutions

$$
u_{i}(x, t)=u_{i}(x \cdot \vec{n}+q t) \text { for } i=1, \ldots, n,
$$

where $\vec{n} \in \mathbb{R}^{d}$ is a chosen unit vector (the direction of propagation) and $q \in \mathbb{R}^{1}$ is the speed of the wave. If we denote $\xi:=x \cdot \vec{n}+q t$, then we arrive at a system of ordinary differential equations of the type:

$$
a_{i}\left(u, u_{i}^{\prime}\right) u_{i}^{\prime \prime}-q c_{i}\left(u, u^{\prime}\right) u_{i}^{\prime}+M_{i}\left(u, u^{\prime}\right) u_{i}^{\prime}+f_{i}(u)=0,
$$

2000 Mathematics Subject Classification. 37B30, 37C29.

Key words and phrases. Reaction diffusion-systems, heteroclinic systems, Conley connection index theory.

This paper was partially supported by grant KBN No 7 T07A 00919. 
where $^{\prime}=d / d \xi, \xi \in \mathbb{R}^{1}$. We are interested in solutions $u(\xi)=\left(u_{1}(\xi), \ldots, u_{n}(\xi)\right)$ such that $\lim _{\xi \rightarrow-\infty}=\mathbf{0}$ and $\lim _{\xi \rightarrow \infty}=\mathbf{1}$, where $\mathbf{0}:=(0, \ldots, 0)$ and $1:=$ $(1, \ldots, 1)$ are stable zeros of the vector function $f=\left(f_{1}, \ldots, f_{n}\right)$. This is a sort of an eigenvalue problem. By this we mean that such solutions may exist only for certain values of $q$. In consequence $q$ is a parameter, which is to be properly chosen.

In this paper we use the Conley connection index theory to prove existence of heteroclinic connections for system (3). The crucial assumption imposed on the coefficients of system (3) consists in the so called local monotonicity of the functions $f_{i}$ (see Assumption 2). Similar problems in the case of constant $a_{i}, c_{i}$ and $M_{i} \equiv 0$ was exhaustively analyzed in the book of Volperts' ([18]). Using the Volperts' methods, Crooks ([6]) extended their results to the case of nonzero $M_{i}$. The method of the existence proof in both [18] and [6] is based on the results in the topological degree theory. There is also a deep paper ([14]) using the alternative method, namely the Conley index theory. However, this paper analyzes slightly different system modelling the behaviour of symbiotic species in ecology, where $f_{i}(u)=u_{i} \tilde{f}(u)$. In the present work we prove the existence of heteroclinic solutions to (3) using also the Conley index theory. The terms $M_{i}$ satisfy more or less the same conditions as in [6], but contrary to [6] the coefficients $a_{i}$ and $c_{i}$ are variable. The general idea of the proof is closely related to the classical paper [4] (and [14]). It should be stressed however that our proofs of auxilliary lemmas take advantage of the results in [18], [6] and [5]. We will be looking for solutions which are strictly monotone, i.e. $u_{i}^{\prime}(\xi)>0, i \in\{1, \ldots, n\}$, for all $\xi \in \mathbb{R}^{1}$. This is a basic assumption, which allows us to choose a proper isolating neighbourhood - a fundamental notion in the Conley index theory.

The paper is organized as follows. Section 1 contains the main assumptions. In Section 2 we define a continuous family of systems depending on a parameter $\lambda \in[0,1]$ coinciding with $(3)$ for $\lambda=1$. In Section 3 we present basic lemmas concerning "a priori" estimates of $\left\|u^{\prime}(\xi)\right\|_{C^{0}\left(\mathbb{R}^{1}\right)}$ and $q$. Let us note that neither the terms $q c_{i}\left(u, u^{\prime}\right) u_{i}^{\prime}$ nor $M_{i}\left(u, u^{\prime}\right) u_{i}^{\prime}$ do not need to grow weaker than $\left|u^{\prime}\right|^{2}$ as $\left|u^{\prime}\right| \rightarrow \infty$ (see Assumptions 3 and 4). In Section 4 we construct a family of isolating neighbourhoods. These neighbourhoods consist approximately of the Cartesian product of the parallelepipeds $[0,1]^{n},[0, m]^{n}$ and $[-Q, Q]$, where $m$ and $Q$ are numbers resulting from "a priori" estimates of the first derivative and the parameter $q$. However, this set is to be properly modified. First, small balls around the limit singular points are added. Secondly, intermediate singular points are to be cut off properly. For some values of the continuation parameter these singular points form a closed subset with nonempty interior of a $n$-dimensional subspace $\left\{(u, z): u \in \mathbb{R}^{n}, z=\mathbf{0}\right\}$. The proof of existence of heteroclinic connections exploits the fact that invariant sets contained in the 
corresponding elements of a continuous family of neighbourhoods have the same Conley index. Thus our task is to make a proper continuation of our problem to the simpler one (i.e. such that can be completely characterized within the terms of the Conley index theory), choose appropriate family of isolating neighbourhoods and to prove that at every step of this continuation the invariant set contained in the closure of the isolating neighbourhood has no common points with its boundary. The main part of our work concerns the systems with the functions $f_{i}, i \in\{1, \ldots, m\}$ satisfying the local monotonicity conditions. In Section 6 we extend our considerations to the case of functions, which can be represented in the form $f_{i}(u)=u_{i} \Phi_{i}\left(u_{i}\right) \tilde{f}_{i}(u)$, with $\tilde{f}_{i}$ satisfying local monotonicity conditions and $\Phi_{i}\left(u_{i}\right)>0$ for $u_{i} \neq 0$ (see Assumtion 5). In Section 7 we prove the existence of heteroclinic connections for a system of equations describing multitemperature plasma sustained by a laser beam.

\section{Main assumptions and auxilliary lemmas}

Assumption 1. All the functions appearing in system (3) are of $C^{1}$-class.

Assumption 2. Assume that

(a) the functions $f_{i} \in C^{1}\left(\mathbb{R}^{n}, \mathbb{R}^{1}\right)$ satisfy the conditions of local monotonicity, i.e. $f_{i, j}(u)>0$ for all $i, j \in\{1, \ldots, n\}, j \neq i$ and all $u \in[0,1]^{n}$ such that $f_{i}(u)=0$.

(b) $\mathbf{0}$ and $\mathbf{1}$ are solutions to the system

$$
f_{i}(u)=0 \quad \text { for } i=1, \ldots, n .
$$

Both constant states $\mathbf{0}$ and $\mathbf{1}$ are stable, i.e. all the eigenvalues of the matrices

$$
f_{i, j}(\mathbf{0}), \quad f_{i, j}(\mathbf{1})
$$

have negative real parts.

(c) All the other solutions $\left\{E_{1}, \ldots, E_{K}\right\}$ to system (4) are contained in $(0,1)^{n}$ and are unstable i.e. the matrix $f_{i, j}\left(E_{k}\right), k=1, \ldots, K$, has at least one eigenvalue with positive real part.

Definition 1. Let $\mathbf{0}=(0, \ldots, 0) \in \mathbb{R}^{n}, \mathbf{1}=(1, \ldots, 1) \in \mathbb{R}^{n},(\mathbf{0}, \mathbf{0})=$ $((0, \ldots, 0),(0, \ldots, 0)) \in \mathbb{R}^{2 n},(\mathbf{1}, \mathbf{0})=((1, \ldots, 1),(0, \ldots, 0)) \in \mathbb{R}^{2 n}$. For any $Y \in \mathbb{R}^{n}$ and natural $m \geq 1$ we put

$$
|Y|=\sup _{i}\left|Y_{i}\right|, \quad \mathbb{R}_{+}^{m}=\left\{y: y \in \mathbb{R}^{m}, y \geq 0\right\} .
$$

For $u, v \in \mathbb{R}^{n}$ we will write $u \geq v(u>v)$, if and only if $u_{i} \geq v_{i}\left(u_{i}>v_{i}\right), i=$ $1, \ldots, n$. 
Assumption 3. $a_{i}\left(u, z_{i}\right)>1$ for all $i \in\{1, \ldots, n\}$, all $\mathbf{0}<u<\mathbf{1}$ and all $z_{i} \in \mathbb{R}_{+}^{1}$. There exists $c_{0}>0$ such that $c_{0}<c_{i}(u, z)$ for all $z \in \mathbb{R}_{+}^{n}, \mathbf{0} \leq u \leq \mathbf{1}$. There exists $b>0$ such that for all $\mathbf{0}<u, v<\mathbf{1}$ and all nonnegative $p$ and $r$ with $|p| \leq|r|$ we have $c_{i}(u, p)\left(c_{i}(v, r)\right)^{-1} \leq b$.

For all $i \in\{1, \ldots, m\}$, let $\chi_{i}: \mathbb{R}_{+}^{1} \rightarrow \mathbb{R}_{+}^{1}$ denote a continuous and increasing function such that

$$
\int_{0}^{z_{i}} \inf _{\mathbf{0} \leq u \leq \mathbf{1}} a_{i}\left(u, z_{i}\right) z_{i} d z_{i} \geq \chi_{i}\left(z_{i}\right) .
$$

Assumption 4. For each $i \in\{1, \ldots, n\}$ one of the below conditions holds:

(a) For all $j \in\{1, \ldots, n\}$ there exist functions $\Gamma_{i j}: \mathbb{R}_{+}^{1} \rightarrow \mathbb{R}_{+}^{1}$ continuous, increasing and such that $\Gamma_{i j}(y) \chi_{i}(y)^{-1} \rightarrow 0$ as $y \rightarrow \infty$, and for all $z_{i} \in \mathbb{R}_{+}^{1}$ :

$$
\int_{0}^{z_{i}} a_{i, j}^{*}\left(z_{i}\right) z_{i} d z_{i} \leq \Gamma_{i j}\left(z_{i}\right),
$$

where $a_{i, j}^{*}\left(z_{i}\right)=\sup _{\mathbf{0} \leq u \leq \mathbf{1}} a_{i, u_{j}}\left(u, z_{i}\right)$. The function $M_{i}(u, z)$ satisfies the inequality

$$
\left|M_{i}(u, z)\right| \leq k(|u|)(1+\beta(|z|)),
$$

with $k: \mathbb{R}_{+}^{1} \rightarrow \mathbb{R}_{+}^{1}$ continuous, and $\beta: \mathbb{R}_{+}^{1} \rightarrow \mathbb{R}_{+}^{1}$ continuous, increasing and such that $\beta(y) y\left(\chi_{i}(y)\right)^{-1} \rightarrow 0$ as $y \rightarrow \infty$.

(b) For all $j \in\{1, \ldots, n\}$ there exist functions $\Gamma_{i j}: \mathbb{R}_{+}^{1} \rightarrow \mathbb{R}_{+}^{1}$ continuous, increasing and such that $\Gamma_{i j}(y) \chi_{i}(y)^{-1} \rightarrow 0$ as $y \rightarrow \infty$ and, for all $z_{i} \in \mathbb{R}_{+}^{1}$

$$
\int_{0}^{z_{i}} a_{* i, j}\left(z_{i}\right) z_{i} d z_{i} \geq-\Gamma_{i j}\left(z_{i}\right)
$$

where $a_{* i, j}\left(z_{i}\right)=\inf _{\mathbf{0} \leq u \leq \mathbf{1}} a_{i, u_{j}}\left(u, z_{i}\right)$, and inequality (7) is satisfied.

(c) $a_{i}\left(u, z_{i}\right)=a_{i}(u)$ and $M_{i}(u, z)$ satisfies condition (7) with $\beta(y) y^{-1} \rightarrow 0$ as $y \rightarrow \infty$ or the sum $a_{i}(u) u_{i}^{\prime \prime}+M_{i}\left(u, u^{\prime}\right) u_{i}^{\prime}$ can be written in the form $\left(a_{i}(u) u_{i}^{\prime}\right)^{\prime}+\mu_{i}\left(u, u^{\prime}\right) u_{i}^{\prime}$ and $\mu_{i}(u, z)$ satisfies (7) with $\beta(y) y^{-1} \rightarrow 0$ as $y \rightarrow \infty$.

(d) $c_{i} \equiv 1$, inequality (6) holds and for all $p, r \in \mathbb{R}_{+}^{n},|p| \leq|r|$,

$$
M_{i}(u, p) \geq M_{i}(v, r)-\widehat{M}_{i}(u, p, v, r)
$$

for all $\mathbf{0} \leq u \leq v \leq \mathbf{1}, \widehat{M}_{i}(u, p, v, r) \leq k(u, v)(1+\beta(|r|))$, with $k$ : $\mathbb{R}_{+}^{2 n} \rightarrow \mathbb{R}_{+}^{1}$ continuous, $\beta: \mathbb{R}_{+}^{1} \rightarrow \mathbb{R}_{+}^{1}$ continuous, increasing and such that $\beta(y) y\left(\chi_{i}(y)\right)^{-1} \rightarrow 0$ as $y \rightarrow \infty$.

(e) $c_{i} \equiv 1$, inequality (8) holds and for all $p, r \in \mathbb{R}_{+}^{n},|p| \leq|r|$,

$$
M_{i}(u, p) \leq M_{i}(v, r)+\widehat{M}_{i}(u, p, v, r)
$$


for all $\mathbf{0} \leq v \leq u \leq \mathbf{1}, \widehat{M}_{i}(u, p, v, r) \leq k(u, v)(1+\beta(|r|))$, with $k$ : $\mathbb{R}_{+}^{2 n} \rightarrow \mathbb{R}_{+}^{1}$ continuous, $\beta: \mathbb{R}_{+}^{1} \rightarrow \mathbb{R}_{+}^{1}$ continuous, increasing and such that $\beta(y) y\left(\chi_{i}(y)\right)^{-1} \rightarrow 0$ as $y \rightarrow \infty$.

REMARK 1. The condition $a_{i}\left(u, z_{i}\right)>1$ in Assumption 3 can be obviously achieved if only $a_{i}\left(u, z_{i}\right)>C_{a i}>0$ for all $z_{i} \in \mathbb{R}_{+}^{1}, \mathbf{0}<u<\mathbf{1}$. The last inequality in Assumption 3 is satisfied for instance if $c_{i}=c_{i}(u)$.

REMARK 2. The condition (a) in Assumption 4 is satisfied for instance if, for all $j \in\{1, \ldots, n\}, a_{i}\left(u, z_{i}\right)_{, u_{j}} \leq 0$ for all $u \in[0,1]^{n}$ and all $z_{i} \in \mathbb{R}_{+}^{1}$ and the condition (b) if $a_{i}\left(u, z_{i}\right)_{, u_{j}} \geq 0$ for all $u \in[0,1]^{n}$ and all $z_{i} \in \mathbb{R}_{+}^{1}$. Let us also note that, when point (c) of Assumption 4 is satisfied, we do not need neither of the conditions (a), (b) to be fulfilled.

Remark 3. Points (d) an (e) of Assumption 4 are approximately the same as in the paper [6]. Let us note that in this case we do not assume any growth condition on the term $M_{i}$.

When dealing with systems satisfying monotonicity conditions the notion of Perron-Frobenius eigenvalue and Perron-Frobenius eigenvector is important. The following lemma will be frequently used below.

Lemma 1 (Perron-Frobenius, see [5, Theorem 1.4]). Let $\mathcal{M}$ be an $n \times n$ matrix with positive off-diagonal elements. Then $\mathcal{M}$ has a real, simple eigenvalue $\mu_{P F}(\mathcal{M})$ such that an associated eigenvector has positive components and every other eigenvalue of $\mathcal{M}$ has real part less than $\mu_{P F}$. Moreover, any positive eigenvector of $\mathcal{M}$ must be a multiple of the eigenvector corresponding to $\mu_{P F}(\mathcal{M}) . \mu_{P F}$ is called the Perron-Frobenius eigenvalue of $\mathcal{M}$, and the associated positive eigenvector $P$ with $\|P\|=1$ the Perron-Frobenius eigenvector of $\mathcal{M}$.

For positive matrices the largest eigenvalue is an increasing function of any of their elements.

Lemma 2 (see [10, Theorem 6, p. 350]). Let $\mathcal{M}$ be an $n \times n$ matrix with positive elements. Then its Perron-Frobenius eigenvalue is a strictly increasing function of any of its entries.

LEMMA 3. Let $\mathcal{M}$ be an $n \times n$ matrix with positive off-diagonal elements. Then its Perron-Frobenius eigenvalue is a strictly increasing function of any of its entries.

Proof. Let us consider the matrix $\mathcal{M}_{c}=\mathcal{M}+c I$, where $I$ is the unit $n \times n$ matrix and $c \in \mathbb{R}^{1}$. Every eigenvalue $\mu$ of $\mathcal{M}$ determines an eigenvalue $\mu_{c}$ of the matrix $\mathcal{M}_{c}$ by the relation

$$
\mu_{c}=c+\mu
$$


If $c$ is taken suffciently large, then all the entries of $\mathcal{M}_{c}$ are positive. Using Lemma 2 and (9) we conlude that the claim of the lemma is true.

\section{Continuation of the system}

We will consider a family of problems depending on the parameter $\lambda \in[0,1]$ :

$$
a_{\lambda i}\left(u, u_{i}^{\prime}\right) u_{i}^{\prime \prime}-J_{\lambda i}\left(q, u, u^{\prime}\right) u_{i}^{\prime}+G_{\lambda i}(u)=0,
$$

where

$$
\begin{aligned}
a_{\lambda i}\left(u, u_{i}^{\prime}\right) & =\psi(\lambda) a_{i}\left(u, u_{i}^{\prime}\right)+(1-\psi(\lambda)), \\
J_{\lambda i}\left(q, u, u^{\prime}\right) u_{i}^{\prime} & =q c_{\lambda i}\left(u, u^{\prime}\right) u_{i}^{\prime}-\psi(\lambda) M_{i}\left(u, u^{\prime}\right) u_{i}^{\prime}, \\
c_{\lambda i}\left(u, u^{\prime}\right) & =\psi(\lambda) c_{i}\left(u, u^{\prime}\right)+(1-\psi(\lambda)) .
\end{aligned}
$$

Here $\psi(\lambda):[0,1] \rightarrow[0,1]$ is a $C^{0,1}$-function such that $\psi \equiv 0$ for $\lambda \in[0,3 / 4]$ and $\psi(\lambda)=4(\lambda-3 / 4)$ for $\lambda \in[3 / 4,1]$. The functions $G_{\lambda i}$ are determined as in the book [18, p. 157] with the parameter $\tau$ in [18] equal to $1-\lambda$. However, for the reader's convenience we sketch this homotopy below. Let $w(s)$ be smooth function of a real variable:

$$
w(s)= \begin{cases}1 & \text { for }|s| \leq \delta / 2, \\ 0 & \text { for }|s| \geq \delta,\end{cases}
$$

such that $w(s)>0$ for $|s|<\delta, \delta \in(0,1 / 2)$. Then we can define the function $\omega(u)$ :

$$
\omega(u)= \begin{cases}w(|u|) & \text { for }|u| \leq \delta \\ w(|u-\mathbf{1}|) & \text { for }|u-\mathbf{1}| \leq \delta \\ 0 & \text { for }|u| \geq \delta,|u-\mathbf{1}| \geq \delta\end{cases}
$$

Let

$$
\omega_{\lambda}(u)=1-3(1-\lambda)+3 \omega(u)(1-\lambda)
$$

for $\lambda \in[2 / 3,1]$. Before proceeding further we introduce an auxilliary function $g: \mathbb{R}^{n} \rightarrow \mathbb{R}^{n}$.

Definition 2. Let $g(u)=\left(g_{1}(u), \ldots, g_{n}(u)\right)$ denote a $C^{1}\left(\mathbb{R}^{n}\right)$-function satisfying for all $i \in\{2, \ldots, n\}$ the following conditions:

(a) $g_{i}\left(u_{1}, u_{2}, \ldots, u_{i-1}, u_{1}, u_{i+1}, \ldots, u_{n}\right)=g_{1}\left(u_{i}, u_{2}, \ldots, u_{i-1}, u_{i}, u_{i+1}, \ldots\right.$, $\left.u_{n}\right)$

(b) $g_{1,1}(u) \leq-k, g_{1, i}(u) \geq k$ for all $u \in \mathbb{R}^{n}, i \in\{2, \ldots, n\}, k>0$.

(c) $\sum_{i=1}^{n} g_{1, i}(\mathbf{0})<0, \sum_{i=1}^{n} g_{1, i}(\mathbf{1})<0$.

(d) the only solutions to the equation $g\left(u_{1}, u_{1}, \ldots, u_{1}\right)=0$ are $\mathbf{0}, \mathbf{1}$ and $E=\left(e_{1}, \ldots, e_{n}\right)$, with $\mathbf{0}<E<\mathbf{1}$ 
For instance, the function $g(u)=\left(g_{1}(u), \ldots, g_{n}(u)\right)$ with $g_{i}(u)=G\left(u_{i}\right)-$ $\sum_{j \neq i} \sigma\left(u_{i}-u_{j}\right)$, where $\sigma>0$ is a sufficiently large constant and $G(y)=-y(y-$ a) $(y-1), a \in(0,1)$, satisfies the above conditions.

REMARK 4. It is easy to show that:

(1) All the solutions to the equation $g(u)=0$ must lie on the diagonal, i.e. all of their components must be equal.

(2) the Perron-Frobenius eigenvalue of $D g(\mathbf{0})$ and $D g(\mathbf{1})$ are negative and equal to $\sum_{i=1}^{n} g_{1, i}(\mathbf{0})$ and $\sum_{i=1}^{n} g_{1, i}(\mathbf{1})<0$ respectively, with the corresponding eigenvectors proportional to $(1, \ldots, 1)$.

Now, let $H$ denote an arbitrary constant matrix with positive off-diagonal elements satisfying the inequalities:

$$
H<D f(\mathbf{0}), D f(\mathbf{1}), D g(\mathbf{0}), D g(\mathbf{1}),
$$

(The inequalities are understood as inequalities between the corresponding entries.)

$$
G_{\lambda}(u)= \begin{cases}f(u) \omega_{\lambda}(u) & \text { for } \lambda \in[2 / 3,1], \\ 3(2 \lambda-1) f(u) \omega(u)+2(2-3 \lambda) h(u) & \text { for } \lambda \in[1 / 2,2 / 3], \\ 3(1-2 \lambda) g(u) \omega(u)+2(3 \lambda-1) h(u) & \text { for } \lambda \in[1 / 3,1 / 2], \\ g(u) \omega_{1-\lambda}(u) & \text { for } \lambda \in[0,1 / 3],\end{cases}
$$

where $h(u)=w(|u|) H u+w(|u-\mathbf{1}|) H(u-\mathbf{1})$.

Thus for $\lambda \in[2 / 3,1]$ the function $G_{\lambda}(u)$ does not change with respect to $\lambda$ for $u$ inside $\delta / 2$ neighbourhoods of the points $\mathbf{0}$ and $\mathbf{1}$ and becomes identically equal to zero outside of $\delta$-neighbourhoods of these points for $\lambda=2 / 3$. For $\lambda \epsilon$ $[1 / 3,2 / 3)$ inside the $\delta$-neighbourhoods the vector function $f(u)$ is homotopically transformed to the function $g(u)$. (It becomes equal to $g(u)$ for $\lambda=1 / 3$.) Finally, for $\lambda \in[0,1 / 3)$, the function $G_{\lambda}(u)$ is transformed to $g(u)$ everywhere in $\mathbb{R}^{n}$. Due to the choice of the matrix $H$ the following lemma holds (see [18, p. 158]).

Lemma 4. For all $\lambda \in[0,1]$ all the eigenvalues of the matrices $D G_{\lambda}(\mathbf{0})$, $D G_{\lambda}(\mathbf{1})$ have negative real parts. For all $\lambda \in[0,1 / 3)$ and $\lambda \in(2 / 3,1]$ the functions $G_{\lambda}(u)$ satisfy the condition of local monotonicity, i.e. $G_{\lambda i, j}(u)>0$ for all $j \neq i$ and all $u \in \mathbb{R}^{n}$ such that $G_{\lambda i}(u)=0$. Moreover, for $\delta>0$ sufficiently small, $G_{\lambda}(u)$ satisfies the condition of local monotonicity for all $\lambda \in[0,1]$ and $u$ such that $|u|<\delta$ or $|u-\mathbf{1}|<\delta$. The only zeros of $G_{\lambda}(u)$ for $|u|<\delta$ or $|u-\mathbf{1}|<\delta$ are $\mathbf{0}$ and $\mathbf{1}$.

Proof. By Assumption 2 and Remark 4 it is obvious that the first statement is true for $\lambda \in[2 / 3,1] \cup[0,1 / 3]$. Note that for $\lambda \in[1 / 2,2 / 3)$ we have $D f(\mathbf{0})>D G_{\lambda}(\mathbf{0}), D f(\mathbf{1})>D G_{\lambda}(\mathbf{1})$ and for $\lambda \in(1 / 3,1 / 2]$ we have $D g(\mathbf{0})>$ 
$D G_{\lambda}(\mathbf{0}), D g(\mathbf{1})>D G_{\lambda}(\mathbf{1})$. Thus by means of Lemma 3 we conclude that the first statement of the lemma is true. The second statement of the lemma follows from the fact that the property of local monotonicity is retained after multiplication by positive functions. While proving the third statement we will examine only the vicinity of the point $\mathbf{0}$. This claim is true for $\lambda \in[2 / 3,1]$. Let us suppose that it is not true for all $\lambda \in[0,2 / 3)$. As the function $\omega(u)>0$ for $|u|<\delta$, this would imply that also the vector function $G_{\omega \lambda}(u)=(\omega(u))^{-1} G_{\lambda}(u)$ has some additional zero for $|u|<\delta$. Hence for some $\widetilde{\lambda} \in[0,2 / 3)$ there would exist $\widetilde{u}, 0<|\widetilde{u}|<\delta$, such that $G_{\omega \widetilde{\lambda}}(\widetilde{u})=0$ and $G_{\omega \lambda}(u) \neq 0$ for all $0<|u|<\delta$ and all $\lambda \in(\widetilde{\lambda}, 1]$. As $f(u), h(u)$ and $g(u)$ are continuously differentiable, then the Perron-Frobenius eigenvalue of the matrix $D G_{\omega \lambda}(u)$ is negative also for all $|u|<\delta$, if $\delta$ is taken sufficiently small. It follows that $\operatorname{det}\left(D G_{\omega \lambda}(u)\right) \neq 0$ for these $u$. In consequence, due to the implicit function theorem, it would follow that for all $\lambda>\widetilde{\lambda}$, but sufficiently close to it, there would exist a continuous branch $u(\lambda)$ such that $u(\lambda) \rightarrow \widetilde{u}$ as $\lambda \rightarrow \widetilde{\lambda}$ and $G_{\omega \lambda}(u(\lambda))=\mathbf{0}$. But this is a contradiction to our supposition. In the same way we consider the vicinity of the point $\mathbf{1}$. The lemma is proved.

Definition 3. A pair $\left(q_{\lambda}, u_{\lambda}\right) \in \mathbb{R}^{1} \times C^{2}\left(\mathbb{R}^{1}, \mathbb{R}^{n}\right), \lambda \in[0,1]$, is called a heteroclinic pair for system (10), if $u_{\lambda}(\xi)$ satisfies system (10) for $q=q_{\lambda}, u_{\lambda}(\xi) \rightarrow \mathbf{0}$ as $\xi \rightarrow-\infty, u_{\lambda}(\xi) \rightarrow \mathbf{1}$ as $\xi \rightarrow \infty$ and $u_{\lambda}^{\prime}(\xi) \rightarrow \mathbf{0}$ as $\xi \rightarrow \pm \infty$. A heteroclinic pair $\left(q_{\lambda}, u_{\lambda}\right)$ is called monotone, if $u_{\lambda}^{\prime}(\xi) \geq 0$ for all $\xi \in \mathbb{R}^{1}$. It is called strictly monotone, if $u_{\lambda}^{\prime}(\xi)>0$ for all $\xi \in \mathbb{R}^{1}$.

The system (10) can be written as a first order system:

$$
\begin{aligned}
& u_{1}^{\prime}=z_{1}, \\
& \ldots \ldots \\
& u_{n}^{\prime}=z_{n} \\
& z_{1}^{\prime}=\left(a_{\lambda i}\left(u, z_{1}\right)\right)^{-1}\left[J_{\lambda 1}(q, u, z) z_{1}-G_{\lambda 1}(u)\right], \\
& \ldots \ldots \ldots \ldots \ldots \ldots \ldots \ldots \ldots \ldots \ldots \ldots \ldots \ldots \ldots \ldots \ldots \ldots \\
& z_{n}^{\prime}=\left(a_{\lambda n}\left(u, z_{n}\right)\right)^{-1}\left[J_{\lambda n}(q, u, z) z_{n}-G_{\lambda n}(u)\right] .
\end{aligned}
$$

To this form of the system (10) we will usually refer, when applying the results of the Conley index theory (e.g. [4], [14]).

\section{A priori estimates of $\left|u^{\prime}\right|_{C^{0}}$ and $q_{\lambda}$}

For monotone solutions to system (10) the following lemma holds.

Lemma 5. Suppose that for $\lambda \in[0,1], q \in \mathbb{R}^{1}, u$ is a bounded $C^{2}\left(\mathbb{R}^{1}\right)$ solution to system $(10)$ such that $\mathbf{0} \leq u(\xi) \leq \mathbf{1}$ and $u^{\prime}(\xi) \geq \mathbf{0}$ for all $\xi \in \mathbb{R}^{1}$. Then there 
exists a constant $m>0$ such that $\left|u^{\prime}\right|_{C^{0}\left(\mathbb{R}^{1}\right)}<m$. This constant is independent of $\lambda \in[0,1], q \in \mathbb{R}^{1}$ and $u$.

Proof. Obviously, $u_{i}^{\prime}(\xi) \rightarrow 0$ as $\xi \rightarrow \pm \infty$, and the $\operatorname{limits} \lim _{\xi \rightarrow \pm \infty} u_{i}(\xi)$ exist for all $i \in\{1, \ldots, n\}$. (In particular we may have $\lim _{\xi \rightarrow-\infty} u(\xi)=\mathbf{0}$ and $\lim _{\xi \rightarrow \infty} u(\xi)=1$.)

Suppose that $\sup _{k} \sup _{\xi \in \mathbb{R}^{1}} u_{k}^{\prime}(\xi)=u_{i}^{\prime}\left(\xi_{0}\right)$. It means that the function $u_{i}^{\prime}(\xi)$ has a maximum at $\xi_{0}$. Thus $u_{i}^{\prime \prime}\left(\xi_{0}\right)=0$. It follows that

$$
q c_{\lambda i}\left(u\left(\xi_{0}\right), u^{\prime}\left(\xi_{0}\right)\right)=\psi(\lambda) M_{i}\left(u\left(\xi_{0}\right), u^{\prime}\left(\xi_{0}\right)\right)+G_{\lambda i}\left(u\left(\xi_{0}\right)\right)\left(u_{i}^{\prime}\left(\xi_{0}\right)\right)^{-1} .
$$

Suppose that condition (a) in Assumption 4 takes place. Let us note that

$$
\begin{aligned}
\int_{-\infty}^{\xi_{0}} & a_{\lambda i}\left(u(s), z_{i}(s)\right) z_{i}(s) z_{i}^{\prime}(s) d s \\
= & \int_{-\infty}^{\xi_{0}}\left\{\left[\int_{0}^{z_{i}(s)} a_{\lambda i}\left(u(s), z_{i}\right) z_{i} d z_{i}\right]_{s}\right. \\
& \left.\quad-\sum_{j} z_{j}(s) \int_{0}^{z_{i}(s)}\left[a_{\lambda i, u_{j}}\left(u(s), z_{i}\right) z_{i} d z_{i}\right]\right\} d s \\
& :=\int_{-\infty}^{\xi_{0}}\left(A_{\lambda i}\left(u(s), z_{i}(s)\right)^{\prime} d s-B_{\lambda i}\left(\xi_{0}\right)=A_{\lambda i}\left(u\left(\xi_{0}\right), z_{i}\left(\xi_{0}\right)\right)-B_{\lambda i}\left(\xi_{0}\right),\right.
\end{aligned}
$$

where, by $A_{\lambda i}\left(u(s), z_{i}\right)$ we have denoted the primitive function of $a_{\lambda i}\left(u(s), z_{i}\right) z_{i}$ with respect to $z_{i}$ such that $A_{\lambda i}(u(s), \mathbf{0})=0$ for all $u(s) \in[0,1]^{n}$. Thus multiplying the $i$-th equation by $u_{i}^{\prime}(\xi)$ and integrating over the interval $\left(-\infty, \xi_{0}\right)$ we obtain:

(18) $A_{\lambda i}\left(u\left(\xi_{0}\right), u_{i}^{\prime}\left(\xi_{0}\right)\right)=B_{\lambda i}\left(\xi_{0}\right)$

$$
\begin{aligned}
& \left.\left.+\int_{-\infty}^{\xi_{0}} q c_{\lambda i}\left(u\left(\xi_{0}\right)\right), u^{\prime}\left(\xi_{0}\right)\right) c_{\lambda i}\left(u(s), u^{\prime}(s)\right)\left[c_{\lambda i}\left(u\left(\xi_{0}\right)\right), u^{\prime}\left(\xi_{0}\right)\right)\right]^{-1} u_{i}^{\prime}(s) u_{i}^{\prime}(s) d s \\
& -\int_{-\infty}^{\xi_{0}} \psi(\lambda) M_{i}\left(u(s), u^{\prime}(s)\right) u_{i}^{\prime}(s) u_{i}^{\prime}(s) d s-\int_{-\infty}^{\xi_{0}} G_{\lambda i}(u(s)) u_{i}^{\prime}(s) d s .
\end{aligned}
$$

Using (16) and Assumption 3 we conclude that the absolute value of the second term at the right hand side of (18) is not greater than:

$$
\begin{aligned}
& \left.\int_{-\infty}^{\xi_{0}} \mid \psi(\lambda) M_{i}\left(u\left(\xi_{0}\right), u^{\prime}\left(\xi_{0}\right)\right)+G_{\lambda i}\left(u\left(\xi_{0}\right)\right)\left(u_{i}^{\prime}\left(\xi_{0}\right)\right)^{-1}\right) \mid \widetilde{b} u_{i}^{\prime}(s) u_{i}^{\prime}(s) d s \\
& \quad \leq \widetilde{b}\left|\psi(\lambda) M_{i}\left(u\left(\xi_{0}\right), u^{\prime}\left(\xi_{0}\right)\right)\right| \int_{-\infty}^{\xi_{0}} u_{i}^{\prime}(s) u_{i}^{\prime}(s) d s+\widetilde{b}\left|G_{\lambda i}\left(u\left(\xi_{0}\right)\right)\right| \int_{-\infty}^{\xi_{0}} u_{i}^{\prime}(s) d s \\
& \quad \leq \widetilde{b}\left|\psi(\lambda) M_{i}\left(u\left(\xi_{0}\right), u^{\prime}\left(\xi_{0}\right)\right)\right| u_{i}^{\prime}\left(\xi_{0}\right) u_{i}\left(\xi_{0}\right)+\widetilde{b}\left|G_{\lambda i}\left(u\left(\xi_{0}\right)\right)\right| u_{i}\left(\xi_{0}\right)
\end{aligned}
$$


where $\widetilde{b}=\max \{1, b\}$ is a number independent of $u_{i}^{\prime}\left(\xi_{0}\right)$. Note that due to the definition of $a_{\lambda i}$ and $\Gamma_{i j}$ we have:

$$
\begin{aligned}
B_{\lambda i}\left(\xi_{0}\right) & \leq \psi(\lambda) \sum_{j} \Gamma_{i j}\left(u_{i}^{\prime}\left(\xi_{0}\right)\right) \int_{-\infty}^{\xi_{0}} u_{j}^{\prime}(s) d s \\
& =\psi(\lambda) \sum_{j} \Gamma_{i j}\left(u_{i}^{\prime}\left(\xi_{0}\right)\right) u_{j}\left(\xi_{0}\right) .
\end{aligned}
$$

Thus, according Assumption 4(a), independently of the value of $q$, we obtain

$$
\begin{aligned}
& (20) \quad \psi(\lambda) \chi_{i}\left(u_{i}^{\prime}\left(\xi_{0}\right)\right)+(1-\psi(\lambda)) \frac{1}{2}\left(u_{i}^{\prime}\left(\xi_{0}\right)\right)^{2} \\
& \leq \quad \psi(\lambda) \sum_{j} u_{j}\left(\xi_{0}\right) \Gamma_{i j}\left(u_{i}^{\prime}\left(\xi_{0}\right)\right)+(1+\widetilde{b}) \psi(\lambda) K\left(1+\beta\left(u_{i}^{\prime}\left(\xi_{0}\right)\right)\right) u_{i}^{\prime}\left(\xi_{0}\right) \\
& \quad+\widetilde{b}\left|G_{\lambda i}\left(u\left(\xi_{0}\right)\right)\right|+\widetilde{G},
\end{aligned}
$$

with $\widetilde{G}$ bounded independently of the solution $u(\xi)$. So, due to the fact that $\beta(y) y(\chi(y))^{-1}=o(1)$ and $\Gamma_{i j}(y)(\chi(y))^{-1}=o(1)$ as $y \rightarrow \infty$, we infer that the value of $u_{i}^{\prime}\left(\xi_{0}\right)$ must be bounded by a number independent $\lambda, q$. This estimation holds for each heteroclinic solution of the system.

Suppose that condition (b) in Assumption 4 takes place. We will use the identity

$$
\begin{aligned}
& \int_{\infty}^{\xi_{0}} a_{\lambda i}\left(u(s), z_{i}(s)\right) z_{i}(s) z_{i}^{\prime}(s) d s \\
&=\int_{\infty}^{\xi_{0}}\left\{\left[\int_{0}^{z_{i}(s)} a_{\lambda i}\left(u(s), z_{i}\right) z_{i} d z_{i}\right]_{s}\right. \\
&\left.-\sum_{j} z_{j}(s) \int_{0}^{z_{i}(s)}\left[a_{\lambda i, u_{j}}\left(u(s), z_{i}\right) z_{i} d z_{i}\right]\right\} d s \\
&= \int_{\infty}^{\xi_{0}}\left(A_{\lambda i}\left(u(s), z_{i}(s)\right)\right)^{\prime} d s-\widetilde{B}_{\lambda i}\left(\xi_{0}\right) \\
&= A_{\lambda i}\left(u\left(\xi_{0}\right), z_{i}\left(\xi_{0}\right)\right)-\widetilde{B}_{\lambda i}\left(\xi_{0}\right) .
\end{aligned}
$$

Thus multiplying the $i$-th equation by $u_{i}^{\prime}(\xi)$ and integrating from $\infty$ up till $\xi$ we obtain the equality similar to (18)

$$
\begin{aligned}
(22) & A_{\lambda i}\left(u\left(\xi_{0}\right), u_{i}^{\prime}\left(\xi_{0}\right)\right)=\widetilde{B}_{\lambda i}\left(\xi_{0}\right) \\
& \left.\left.+\int_{\infty}^{\xi_{0}} q c_{\lambda i}\left(u\left(\xi_{0}\right)\right), u^{\prime}\left(\xi_{0}\right)\right) c_{\lambda i}\left(u(s), u^{\prime}(s)\right)\left[c_{\lambda i}\left(u\left(\xi_{0}\right)\right), u^{\prime}\left(\xi_{0}\right)\right)\right]^{-1} u_{i}^{\prime}(s) u_{i}^{\prime}(s) d s \\
& -\int_{\infty}^{\xi_{0}} \psi(\lambda) M_{i}\left(u(s), u^{\prime}(s)\right) u_{i}^{\prime}(s) u_{i}^{\prime}(s) d s-\int_{\infty}^{\xi_{0}} G_{\lambda i}(u(s)) u_{i}^{\prime}(s) d s .
\end{aligned}
$$


Due to the fact that we integrate from $\infty$ to $\xi$ we obtain, by using the condition (b) in Assumption 4, (16) and Assumption 3, similarly as above:

$$
\begin{aligned}
& \psi(\lambda) \chi_{i}\left(u_{i}^{\prime}\left(\xi_{0}\right)\right)+(1-\psi(\lambda)) \frac{1}{2}\left(u_{i}^{\prime}\left(\xi_{0}\right)\right)^{2} \\
& \leq \psi(\lambda) \sum_{j}\left(1-u_{j}\left(\xi_{0}\right)\right) \Gamma_{i j}\left(u_{i}^{\prime}\left(\xi_{0}\right)\right)+\widetilde{b} \psi(\lambda)\left|M_{i}\left(u\left(\xi_{0}\right), u^{\prime}\left(\xi_{0}\right)\right)\right| u_{i}^{\prime}\left(\xi_{0}\right)\left(1-u_{i}\left(\xi_{0}\right)\right) \\
& \quad+(1+\widetilde{b}) \psi(\lambda) K\left(1+\beta\left(u_{i}^{\prime}\left(\xi_{0}\right)\right)\right) u_{i}^{\prime}\left(\xi_{0}\right)\left(1-u_{i}\left(\xi_{0}\right)\right)+\widetilde{b}\left|G_{\lambda i}\left(u\left(\xi_{0}\right)\right)\right|+\underline{G},
\end{aligned}
$$

where $\widetilde{b}=\max \{1, b\}$ is a number independent of $u_{i}^{\prime}\left(\xi_{0}\right)$ and $\underline{G}$ is bounded independently of the particular solution $u(\xi)$. So, due to the fact that

$$
\beta(y) y(\chi(y))^{-1}=o(1) \quad \text { and } \quad \Gamma_{i j}(y)(\chi(y))^{-1}=o(1)
$$

as $y \rightarrow \infty$, we infer that the value of $u_{i}^{\prime}\left(\xi_{0}\right)$ must be bounded by a number independent $\lambda, q$ and a particular heteroclinic solution of the system.

When Assumption 4(c) is fulfilled, the proof may be carried out similarly. First, when $M_{i}(u, z)$ satisfies (7), we can divide the $i$-th equation by $a_{i}$ and obtain the equation with the coefficient by $u_{i}^{\prime \prime}$ equal to 1 . (After this operation Assumptions 2 and 3 retain their validity). Suppose that the second possibility of point (c) holds. Multiplying the $i$-th equation by $a_{\lambda i}(u(s)) u_{i}^{\prime}(s)$ and integrating from $-\infty$ to $\xi_{0}$ we obtain the equality

$$
\begin{aligned}
\frac{1}{2}\left[a_{\lambda i}\left(u\left(\xi_{0}\right)\right) u_{i}^{\prime}\left(\xi_{0}\right)\right]^{2}= & \int_{-\infty}^{\xi_{0}} q c_{\lambda i}\left(u(s), u^{\prime}(s)\right) a_{\lambda i}(u(s)) u_{i}^{\prime}(s) u_{i}^{\prime}(s) d s \\
& -\int_{-\infty}^{\xi_{0}} \psi(\lambda) \mu_{i}\left(u(s), u^{\prime}(s)\right) a_{\lambda i}(u(s)) u_{i}^{\prime}(s) u_{i}^{\prime}(s) d s \\
& -\int_{-\infty}^{\xi_{0}} G_{\lambda i}(u(s)) a_{\lambda i}(u(s)) u_{i}^{\prime}(s) d s,
\end{aligned}
$$

from which one may obtain the desired estimate.

Suppose that Assumption 4(d) is satisfied. We have as before

$$
q=\psi(\lambda) M_{i}\left(u\left(\xi_{0}\right), u^{\prime}\left(\xi_{0}\right)\right)+G_{\lambda i}\left(u\left(\xi_{0}\right)\right)\left(u_{i}^{\prime}\left(\xi_{0}\right)\right)^{-1} .
$$

Then, as above, we obtain

$$
\begin{aligned}
\chi_{i}\left(u_{i}^{\prime}\left(\xi_{0}\right)\right)+(1-\psi(\lambda)) \frac{1}{2}\left(u_{i}^{\prime}\left(\xi_{0}\right)\right)^{2} \\
\leq B_{\lambda i}\left(\xi_{0}\right)+\int_{-\infty}^{\xi_{0}}\left(q-\psi(\lambda) M_{i}\left(u(s), u^{\prime}(s)\right) u_{i}^{\prime}(s) u_{i}^{\prime}(s) d s\right. \\
\quad-\int_{-\infty}^{\xi_{0}} G_{\lambda i}(u(s)) u_{i}^{\prime}(s) d s \\
\quad \leq \int_{-\infty}^{\xi_{0}}\left[q-\psi(\lambda) M_{i}\left(u\left(\xi_{0}\right), u^{\prime}\left(\xi_{0}\right)\right)-G_{\lambda i}\left(u\left(\xi_{0}\right)\right)\left(u_{i}^{\prime}\left(\xi_{0}\right)\right)^{-1}\right] u_{i}^{\prime}(s) u_{i}^{\prime}(s) d s
\end{aligned}
$$




$$
\begin{gathered}
\left.\quad+\int_{-\infty}^{\xi_{0}} G_{\lambda i}\left(u\left(\xi_{0}\right)\right)\left(u_{i}^{\prime}\left(\xi_{0}\right)\right)^{-1}\right) u_{i}^{\prime}(s) u_{i}^{\prime}(s) d s-\int_{-\infty}^{\xi_{0}} G_{\lambda i}(u(s)) u_{i}^{\prime}(s) d s \\
+\psi(\lambda) \int_{-\infty}^{\xi_{0}}\left[M_{i}\left(u\left(\xi_{0}\right), u^{\prime}\left(\xi_{0}\right)\right)-M_{i}\left(u(s), u^{\prime}(s)\right)\right] u_{i}^{\prime}(s) u_{i}^{\prime}(s) d s+B_{\lambda i}\left(\xi_{0}\right) \\
\leq C_{i}+\psi(\lambda) K\left(1+\beta\left(u_{i}^{\prime}\left(\xi_{0}\right)\right)\right) u_{i}^{\prime}\left(\xi_{0}\right) u_{i}\left(\xi_{0}\right)+\psi(\lambda) \sum_{j} u_{j}\left(\xi_{0}\right) \Gamma_{i j}\left(u_{i}^{\prime}\left(\xi_{0}\right)\right),
\end{gathered}
$$

where $C_{i}$ and $K$ are independent of $u_{i}^{\prime}\left(\xi_{0}\right)$. Hence, as before we conclude that the lemma is true.

When Assumption 4(e) is satisfied then we integrate from $\infty$ up till $\xi_{0}$. We obtain:

$$
\begin{aligned}
\chi_{i}\left(u_{i}^{\prime}\left(\xi_{0}\right)\right)+(1-\psi(\lambda)) \frac{1}{2}\left(u_{i}^{\prime}\left(\xi_{0}\right)\right)^{2} \\
\leq \widetilde{B}_{\lambda i}\left(\xi_{0}\right)+\int_{\infty}^{\xi_{0}}\left[q-\psi(\lambda) M_{i}\left(u\left(\xi_{0}\right), u^{\prime}\left(\xi_{0}\right)\right)\right. \\
\left.\quad-G_{\lambda i}\left(u\left(\xi_{0}\right)\right)\left(u_{i}^{\prime}\left(\xi_{0}\right)\right)^{-1}\right] u_{i}^{\prime}(s) u_{i}^{\prime}(s) d s \\
\left.\quad+\int_{\infty}^{\xi_{0}} G_{\lambda i}\left(u\left(\xi_{0}\right)\right)\left(u_{i}^{\prime}\left(\xi_{0}\right)\right)^{-1}\right) u_{i}^{\prime}(s) u_{i}^{\prime}(s) d s-\int_{\infty}^{\xi_{0}} G_{\lambda i}(u(s)) u_{i}^{\prime}(s) d s \\
\quad+\psi(\lambda) \int_{\infty}^{\xi_{0}}\left[M_{i}\left(u\left(\xi_{0}\right), u^{\prime}\left(\xi_{0}\right)\right)-M_{i}\left(u(s), u^{\prime}(s)\right)\right] u_{i}^{\prime}(s) u_{i}^{\prime}(s) d s \\
\leq C_{i}+\psi(\lambda) K\left(1+\beta\left(u_{i}^{\prime}\left(\xi_{0}\right)\right)\right) u_{i}^{\prime}\left(\xi_{0}\right)\left(1-u_{i}\left(\xi_{0}\right)\right) \\
\quad+\psi(\lambda) \sum_{j}\left(1-u_{j}\left(\xi_{0}\right)\right) \Gamma_{i j}\left(u_{i}^{\prime}\left(\xi_{0}\right)\right),
\end{aligned}
$$

where $C_{i}$ and $K$ are independent of $u_{i}^{\prime}\left(\xi_{0}\right)$. The lemma is proved.

The following lemma holds.

Lemma 6. Let the components of $F \in C^{1}\left(\mathbb{R}^{n}, \mathbb{R}^{n}\right)$ satisfy Assumption 2. Let $P(\mathbf{0})$ and $P(\mathbf{1})$ denote the eigenvector corresponding to the Perron-Frobenius eigenvalue of $D F(\mathbf{0})$ and $D F(\mathbf{1})$, respectively. Then there exist $r>0$ and $\vartheta>0$ such that for each $i \in\{1, \ldots, n\}$

$$
\begin{aligned}
& \operatorname{dist}\left(u, W_{0 i}\right)<\vartheta(r) \Rightarrow F_{i}(u)<0, \\
& \operatorname{dist}\left(u, W_{1 i}\right)<\vartheta(r) \Rightarrow F_{i}(u)>0,
\end{aligned}
$$

where

$$
\begin{aligned}
& W_{0 i}=\left\{u: \mathbf{0} \leq u \leq r P(\mathbf{0}), u_{i}=r P_{i}(\mathbf{0})\right\}, \\
& W_{1 i}=\left\{u: \mathbf{1} \geq u \geq r P(\mathbf{1}), u_{i}=1-r P_{i}(\mathbf{1})\right\} .
\end{aligned}
$$

Proof. Let $\mu_{F}$ denote the Perron-Frobenius eigenvalue of $D F(\mathbf{0})$. Then $r$ may be taken so small that $F(r P(\mathbf{0}))=r D F P(\mathbf{0})+o(r)<r \mu_{F} P(\mathbf{0}) / 2$. Moreover, we can decrease $r$ if necessary, so that the monotonicity conditions from 
Assumption 2(a) hold in the set $\mathbf{0} \leq u \leq 2 r P(\mathbf{0})$. Using these conditions we conclude that $F_{i}(u)<0$ for $u \in W_{0 i}$. In consequence there exists $\vartheta=\vartheta(r)>0$ such that the first of the above relations is satisfied. In the same way we prove the second relation.

LEMma 7. Let $F$ and $r$ be the same as in Lemma 6. Then for any point $u \in W_{0}=\{u: \mathbf{0} \leq u \leq r P(\mathbf{0}), u \neq \mathbf{0}\}$ there exists $i \in\{1, \ldots, n\}$ such that $F_{i}(u)<0$. Likewise, for any point $u \in W_{1}=\{u: \mathbf{1} \geq u \geq \mathbf{1}-r P(\mathbf{1}), u \neq \mathbf{1}\}$ there exists $i \in\{1, \ldots, n\}$ such that $F_{i}(u)>0$.

Proof. Let us take an arbitrary point $U=\left(U_{1}, \ldots, U_{n}\right) \in W_{0}$. Let $\widetilde{r}=$ $\max _{j} U_{j}\left(r P_{j}(\mathbf{0})\right)^{-1}=U_{k}\left(r P_{k}(\mathbf{0})\right)^{-1}$ for some $k \in\{1, \ldots, n\}$. As $\widetilde{r} \leq r$ then it follows from the proof of Lemma 6 that it holds with $r$ replaced by $\widetilde{r}$ and $\vartheta(r)$ replaced by $\vartheta(\widetilde{r})$. In consequence $F_{k}(u)<0$ for $u \in \widetilde{W}_{0 k}=\{u: \mathbf{0} \leq u \leq$ $\left.\widetilde{r} P(\mathbf{0}), u_{k}=\widetilde{r} P_{k}(\mathbf{0})\right\}$. In the same way we consider the parallelepiped $W_{1}$. The lemma is proved.

Another proof of Lemma 7 can be found in [18, p. 159].

As a corollary to Lemma 7 we have the following lemma.

Lemma 8. Let $F$ be the same as in Lemma 6. Then there does not exist a point $u, 0<|u|<\widetilde{\delta}, \widetilde{\delta}$ sufficiently small, such that $F_{i}(u) \geq 0$ for all $i \in$ $\{1, \ldots, n\}$. Likewise there does not exist a point $u, 0<|\mathbf{1}-u|<\widetilde{\delta}, \widetilde{\delta}$ sufficiently small, such that $F_{i}(u) \leq 0$ for all $i \in\{1, \ldots, n\}$.

Proof. It suffices to take $\widetilde{\delta}<r$ and apply Lemma 7 .

REMARK 5. It is easy to note that if $r$ and $\widetilde{\delta}$ are taken sufficiently small then Lemmas 6-8 hold also for the functions $G_{\lambda}, \lambda \in[0,1]$.

Now, we are able to prove a priori estimates for $q$.

LEMMA 9. If $\left(q_{\lambda}, u_{\lambda}\right)$ is a strictly monotone heteroclinic pair for system (10), then $\left|q_{\lambda}\right|<Q$, where $Q$ is independent of $\lambda \in[0,1]$ and $u_{\lambda}$.

Proof. For simplicity, we will omit the subscript $\lambda$ in $q_{\lambda}$ and $u_{\lambda}$. The idea of the proof is contained in [6] (Lemma 3.4). As $u(\xi) \rightarrow \mathbf{0}$ monotonically as $\xi \rightarrow-\infty$, then there must exist an index $i$ and $\xi=\xi_{0}$ such that $u(\xi)$ enters the region $\mathbf{0} \leq u \leq r P(\mathbf{0})$ through the (closed) set $W_{0 i}$, i.e. $u\left(\xi_{0}\right) \in W_{0 i}$ (see Lemma 6). Let us take $\xi_{1}<\xi_{0}$ such that $u_{i}\left(\xi_{0}\right)-u_{i}\left(\xi_{1}\right)=\vartheta / 2$. Integrating the $i$-th equation of the system (10) we obtain

$$
\begin{aligned}
\int_{\xi_{1}}^{\xi_{0}} a_{\lambda i}\left(u(s), u_{i}^{\prime}(s)\right) u_{i}^{\prime \prime}(s) d s-\int_{\xi_{1}}^{\xi_{0}} c_{\lambda i}\left(u(s), u^{\prime}(s)\right) q u_{i}^{\prime}(s) d s \\
-\int_{\xi_{1}}^{\xi_{0}} \psi(\lambda) M_{i}\left(u(s), u^{\prime}(s)\right) u_{i}^{\prime}(s) d s+\int_{\xi_{1}}^{\xi_{0}} G_{\lambda i}(u(s)) d s=0 .
\end{aligned}
$$


Let us note that

$$
\begin{aligned}
\int_{\xi_{1}}^{\xi_{0}} a_{\lambda i}\left(u(s), u_{i}^{\prime}(s)\right) u_{i}^{\prime \prime}(s) d s= & \widetilde{A}_{\lambda i}\left(u\left(\xi_{1}\right), u_{i}^{\prime}\left(\xi_{1}\right)\right)-\widetilde{A}_{\lambda i}\left(u\left(\xi_{0}\right), u_{i}^{\prime}\left(\xi_{0}\right)\right) \\
& +\int_{\xi_{1}}^{\xi_{0}} \sum_{j} z_{j}(s) \int_{0}^{z_{i}(s)}\left[a_{\lambda i, u_{j}}\left(u(s), z_{i}\right) d z_{i}\right] d s,
\end{aligned}
$$

where $\widetilde{A}_{\lambda i}\left(u(s), z_{i}\right)$ is the primitive function of $a_{\lambda i}\left(u(s), z_{i}\right)$ such that $\widetilde{A}_{\lambda i}(u(s), \mathbf{0})$ $=0$. The expressions on the right hand side are bounded, due to the Lemma 5 , so $\int_{\xi_{1}}^{\xi_{0}} a_{\lambda i}\left(u^{\prime}(s)\right) u_{i}^{\prime \prime}(s) d s<R_{1}<\infty$. The third term on the left hand side of equation (23) is also bounded from above independently of $\lambda, \xi_{0}$ and $\xi_{1}$ by some finite number $R_{2}$. If $\left(R_{1}+R_{2}\right) \leq 0$, then $q$ is negative, due to the fact that $\int_{\xi_{1}}^{\xi_{0}} G_{\lambda i}(u(s)) d s<0$. So, suppose that $\left(R_{1}+R_{2}\right)>0$ and $q \geq 0$. Then, in view of Assumption 3, the second term can be estimated as follows:

$$
\begin{aligned}
\int_{\xi_{1}}^{\xi_{0}} c_{\lambda i}\left(u(s), u^{\prime}(s)\right) q u_{i}^{\prime}(s) d s & \geq q c_{0} \int_{\xi_{1}}^{\xi_{0}} u_{i}^{\prime}(s) d s \\
& =q c_{0} \int_{\xi_{1}}^{\xi_{0}}\left(u_{i}\left(\xi_{0}\right)-u_{i}\left(\xi_{1}\right)\right) \geq q c_{0} \frac{\vartheta}{2} .
\end{aligned}
$$

Consequently, from (23) we obtain the relation $q c_{0} \vartheta<2\left(R_{1}+R_{2}\right)$, which implies that $q<c_{0}^{-1} \vartheta^{-1} 2\left(R_{1}+R_{2}\right)$. In the same way, by analyzing the behaviour of a heteroclinic trajectory near the singular point $(\mathbf{1}, \mathbf{0})$ we can prove the boundedness of the parameter $q$ from below.

\section{Construction of isolating neighbourhoods}

For the notions concerning the Conley and connection index theory we refer the reader to [2], [4], [13] and [17]. The a priori estimates of the previous section allow us to find an isolating neighbourhood, i.e. a compact set having the property that no invariant set contained in it touches its boundary. The isolating neighbourhood will be defined in several steps. Let

$$
\begin{aligned}
N & =\left\{(u, z): u_{i} \in[0,1], z_{i} \in[0, m], i \in\{1, \ldots, n\}\right\}, \\
N^{*}(\varepsilon) & =N \cup \mathcal{B}(A, \varepsilon) \cup \mathcal{B}(B, \varepsilon),
\end{aligned}
$$

where $m$ is a number given by Lemma 5 and $\mathcal{B}(A, \varepsilon)$ and $\mathcal{B}(B, \varepsilon)$ denote the closed balls in the space $(u, z)$ of radius $\varepsilon$ with centers at the points $A=(\mathbf{0}, \mathbf{0})$ and $B=(\mathbf{1}, \mathbf{0})$, respectively. Explicitly

$$
\begin{aligned}
& \mathcal{B}(A, \varepsilon)=\{(u, z): \sup \{|u|,|z|\} \leq \varepsilon\}, \\
& \mathcal{B}(B, \varepsilon)=\{(u, z): \sup \{|\mathbf{1}-u|,|z|\} \leq \varepsilon\} .
\end{aligned}
$$

The number $\varepsilon$ must be taken sufficiently small. Its upper value will be determined in a series of lemmas below. 
Lemma 10. For each $\lambda \in[0,1]$ and each $q \in[-Q, Q]$ the points $A=(\mathbf{0}, \mathbf{0})$ and $B=(\mathbf{1}, \mathbf{0})$ are saddle singular points of system (15). The linearization matrices of system (15) at these points have exactly $n$ eigenvalues in the left half plane and $n$ eigenvalues in the right half plane.

Proof. See Theorem 3.3 in [5].

Having an arbitrary compact subset $\mathcal{N}$ in the phase space $(u, z)$ by $I(\mathcal{N})$ we will understand the maximal invariant set contained in $\mathcal{N}$ i.e. the set of all points $\left(u_{0}, z_{0}\right) \in \mathcal{N}$ such that the trajectory of a solution $(u(\xi), z(\xi)), \xi \in \mathbb{R}^{1}$, to system (15) satisfying initial condition $(u(0), z(0))=\left(u_{0}, z_{0}\right)$ lies in $\mathcal{N}$.

First we will show that if a trajectory belongs to $I\left(N^{*}(\varepsilon)\right)$, then it cannot leave the set $N$ if only $\varepsilon$ is taken sufficiently small.

Lemma 11. For $q \in[-Q, Q]$ with $Q$ as in Lemma 9 and all $\varepsilon \in\left(0, \varepsilon_{0}\right)$ with $\varepsilon_{0}>0$ sufficiently small no trajectory of a solution to system (10) belonging to $I\left(N^{*}\right)$ can enter the set $N^{*} \backslash N$.

Proof. We consider only the ball $\mathcal{B}(B, \varepsilon)$. As $B$ is a saddle singular point then any trajectory leaves the ball $\mathcal{B}(B, \varepsilon)$ in forward or backward direction, if only $\varepsilon$ is taken sufficiently small ( $\varepsilon$ can be taken independently of $\lambda \in[0,1]$ and $q \in[-Q, Q]$ ). According to the Hartman-Grobman theorem (see e.g. [1]) sufficiently close to the saddle singular point the flow generated by our system is $C^{0}$-equivalent to the flow generated by its linearization. It follows that for $\xi \rightarrow \infty$ or $\xi \rightarrow-\infty$ the considered trajectory either leaves the ball $\mathcal{B}(B, \varepsilon)$ or tends to the point $B$ if $\varepsilon>0$ is small enough. So, suppose that a trajectory from $I\left(N^{*}\right)$ leaves the set $N$ and enters the set $\mathcal{B}(B, \varepsilon) \backslash N$. We will prove that this trajectory can neither reach the point $B$ nor enter the set $N$ again. Suppose that this trajectory reaches the point $B$ without entering the set $N$ again. It is easy to note that then there must exist an index $i$ and numbers $\xi_{1} \in \mathbb{R}^{1}, \delta>0$, such that $u_{i}^{\prime}(\xi)>0$ for $\xi \in\left(\xi_{1}-\delta, \xi_{1}\right), u_{i}^{\prime}\left(\xi_{1}\right)=0$, and such that $u_{i}^{\prime}(\xi)<0$ for $\xi \in\left(\xi_{1}, \xi_{1}+\delta\right)$. Moreover, $u_{i}^{\prime}(\xi)$ must have a global minimum in the set $\left(\xi_{1}, \infty\right)$. Due to the fact that $u^{\prime}(\xi) \rightarrow \mathbf{0}$ as $\xi \rightarrow \infty$ for all $j$ the quantity $\inf _{\xi \in\left(\xi_{1}, \infty\right)} u_{j}^{\prime}(\xi)$ is finite and at least for $j=i$ it is negative. Thus there exists a unique $s>0$ such that the function $v_{s}(\xi)=u^{\prime}(\xi)+s P_{\lambda}(\mathbf{1}):=v(\xi)+s P_{\lambda}(\mathbf{1}) \geq \mathbf{0}$ for all $\xi \in\left(\xi_{1}, \infty\right)$ and such that for some $\xi^{*} \in\left(\xi_{1}, \infty\right)$ the vector $v_{s}\left(\xi^{*}\right)$ has at least one zero component. Here $P_{\lambda}(\mathbf{1})$ is the eigenvector corresponding to the PerronFrobenius eigenvalue of the matrix $D G_{\lambda}(\mathbf{1})$. To fix our attention suppose that $v_{s 1}\left(\xi^{*}\right)=0$. Thus $v_{1}\left(\xi^{*}\right)=-s P_{\lambda 1}(\mathbf{1}), v_{1}^{\prime}\left(\xi^{*}\right)=0$ and $v_{1}^{\prime \prime}\left(\xi^{*}\right) \geq 0$. Let us note that the components of $v(\xi)$ satisfy the equation

$$
a_{\lambda i}\left(u, u_{i}^{\prime}\right) v_{i}^{\prime \prime}+T_{i}\left(\lambda, q, u, u^{\prime}\right) v_{i}^{\prime}+\sum_{j}\left\{G_{\lambda i, j}(u)+S_{\lambda i}\left(\lambda, q, u, u^{\prime}\right) \delta_{i j}\right\} v_{j}=0,
$$


obtained by differentiation and expressing $u_{j}^{\prime \prime}, j \neq i$, from the $j$-th equation of system (10). We note that the function $S_{\lambda i}$ behaves like $O\left(\left|u^{\prime}(\xi)\right|\right)+O(|\mathbf{1}-u(\xi)|)$ as $\xi \rightarrow \infty$. Let $\mathcal{K}\left(\lambda, q, u, u^{\prime}\right)$ denote the matrix with the entries $\mathcal{K}_{i j}$ equal to the expressions in the braces in (24). Let $\varepsilon_{0}<\delta / 2$ be so small that in the set $\left\{(u, z): \sup \{|\mathbf{1}-u|,|z|\}<\varepsilon_{0}\right\}$, independently of $\lambda \in[0,1]$ and $q \in[-Q, Q]$, the following conditions are satisfied:

(1) $\mathcal{K}(\lambda, q, u, z)$ has all of its off-diagonal terms positive,

(2) $\mathcal{K}(\lambda, q, u, z) P_{\lambda}(\mathbf{1})<\mathbf{0}$.

It is obvious that the constant $\varepsilon_{0}$ with the above mentioned properties exists. Now, we will show that if $\sup \left\{\left|\mathbf{1}-u\left(\xi^{*}\right)\right|,\left|u^{\prime}\left(\xi^{*}\right)\right|\right\}<\varepsilon_{0}$ then

$$
\sum_{j}\left\{G_{\lambda 1, j}\left(u\left(\xi^{*}\right)\right)+S_{\lambda 1}\left(\lambda, q, u\left(\xi^{*}\right), u^{\prime}\left(\xi^{*}\right)\right) \delta_{1 j}\right\} v_{j}\left(\xi^{*}\right)>0 .
$$

To prove it let us consider the function

$$
\phi(y)=\left\{\sum_{j}\left(G_{\lambda 1, j}(u)+S_{\lambda 1}\left(\lambda, q, u, u^{\prime}\right) \delta_{1 j}\right\}\left(\xi^{*}\right)\left(v_{j}\left(\xi^{*}\right)(1-y)-s P_{\lambda j}(\mathbf{1}) y\right) .\right.
$$

If $v\left(\xi^{*}\right)=-s P_{\lambda}(\mathbf{1})$, the claim results from the assumption imposed on $\varepsilon_{0}$. If $v\left(\xi^{*}\right) \neq-s P_{\lambda}(\mathbf{1})$, then for at least one $j \neq i$ we have $v_{j}\left(\xi^{*}\right)-s P_{\lambda j}(\mathbf{1})>0$, so $\phi^{\prime}(y)<0$ (due to the fact that $\mathcal{K}\left(\lambda, q, u, u^{\prime}\right.$ ) has all of its off-diagonal terms positive inside the ball $\mathcal{B}(B, \varepsilon)), \phi(1)>0$, hence $\phi(0)>0$. Thus inequality (25) is proved. But this contradicts (24) for $i=1$ as $v_{1}^{\prime}\left(\xi^{*}\right)=0$ and $v_{1}^{\prime \prime}\left(\xi^{*}\right) \geq 0$. Now, suppose that the considered trajectory enters the set $N$ again. It means that there exists $\xi_{2} \in\left(\xi_{1}, \infty\right)$ such that $u^{\prime}\left(\xi_{2}\right) \geq 0$. Consequently, the whole proof may be repeated verbatim. (This time $\xi^{*} \in\left(\xi_{1}, \xi_{2}\right)$.)

REMARK 6. The first part of the proof of Lemma 11 can be carried out without using the Hartman-Grobman theorem. We will prove it near the point $A$. Near the point $B$ the proof is the same. Suppose first, that for an invariant trajectory we have $u_{i}\left(\xi_{0}\right)<0$ for some index $i$ and some finite $\xi_{0}$. Suppose that $z_{i}\left(\xi_{0}\right)>0$. By considering the backward trajectory we infer that either $z_{i}\left(\xi_{0}\right)$ becomes negative for some $\xi<\xi_{0}$ or at least $z_{i}(\xi) \rightarrow 0$ as $\xi \rightarrow-\infty$. In the latter case $u_{i}(-\infty)<0$. If we assume that $z_{j}(\xi) \geq 0$ for all $\xi \in \mathbb{R}^{1}$ and all $j \neq i$, then, as $\xi \rightarrow-\infty$ we have $z_{k}(\xi) \rightarrow 0$ for all $k \in\{1, \ldots, n\}$ and the limits $\lim _{\xi \rightarrow-\infty} u_{k}(\xi)$ exist. However, as $u_{i}(-\infty)<0$, then at least one component of $G_{\lambda}\left(u(-\infty)\right.$ ), say $G_{\lambda l}(u(-\infty)) \neq 0$. Hence $z_{l}^{\prime}(\xi)$ tends (as $\left.\xi \rightarrow-\infty\right)$ to a number, which is different from 0 . But this contradicts the fact that $z_{l}(\xi) \rightarrow 0$. Similar conclusion holds if $z_{i}\left(\xi_{0}\right)=0$. As a result, for at least one $j \in\{1, \ldots, n\}$ we must have $z_{j}\left(\xi_{*}\right)<0$ for some finite $\xi_{*}$. The same is obviously true if an invariant trajectory leaves the set $z \geq 0$ inside $\mathcal{B}(A, \varepsilon)$. As the solutions inside $N^{*}$ are bounded then the function $z_{j}$ must attain a negative minimum for some finite $\xi$ 
inside $\mathcal{B}(A, \varepsilon)$. Now, the rest of the proof may be carried out similarly to the proof of Lemma 11. It is seen that with this modification Lemma 11 is valid also in the case, when $q$ is not a constant parameter, but changes suffficiently slowly. Thus suppose that $q^{\prime}=\rho \phi(u, z) q$, where $\phi$ is globally bounded and $\rho>0$ is a parameter, which can be taken arbitrarily small. Then the matrix $\mathcal{K}(\lambda, q, u, z)$ changes to $\widetilde{\mathcal{K}}(\lambda, q, u, z)=\mathcal{K}(\lambda, q, u, z)-\rho q c_{\lambda i}(u, z) \phi(u, z) \delta_{i j}$. It is obvious that for $|\rho|$ sufficiently small $\widetilde{\mathcal{K}}$ satisfies the conditions (1) and (2) fulfilled by the matrix $\mathcal{K}$ (at the point $\mathbf{0}$ ). (Condition (1) is obviously satisfied. Due to the fact that $\lambda$ and $q$ belong to the compact sets we have $\mathcal{K}(\lambda, q, u, z) P_{\lambda}(\mathbf{0})<k<\mathbf{0}$, independently of $\lambda$ and $q$, hence for $|\rho|$ sufficiently small we have $\widetilde{\mathcal{K}}(\lambda, q, u, z) P_{\lambda}(\mathbf{0})<\mathbf{0}$.) The rest of the proof may be repeated without changes.

It follows from Lemma 11 that $I\left(N^{*}\right)=I(N)$, thus for any trajectory from $I\left(N^{*}\right)$ we have $u_{i}(\xi) \geq 0$ for all $\xi \in \mathbb{R}^{1}$. One notes that for $\lambda \in(2 / 3,1] \cup[0,1 / 3)$ and all $q \in[-Q, Q]$ the boundary of $N^{*}$ contains at least intermediate singular points belonging to $I\left(N^{*}\right)$. For other values of the parameter $\lambda$ the situation may be even more complicated. To use the Conley connection index theory we must analyze the structure of the set $I(N) \cap \partial N$ and to modify the set $N^{*}$ in such a way that it becomes an isolating neighbourhood.

First, we will analyze the set $I(N) \cap \partial N$ for $\lambda \in(2 / 3,1] \cup[0,1 / 3)$. As we noted the following lemma holds.

Lemma 12. For $\lambda \in(2 / 3,1] \cup[0,1 / 3)$ and $q \in[-Q, Q]$

$$
I(N) \cap \partial N=\left\{(u, \mathbf{0}), u \in[0,1]^{n}: G_{\lambda}(u)=\mathbf{0}\right\} .
$$

REMARK 7. Due to the form of $G_{\lambda}$, the last set does not change on each of the intervals $(2 / 3,1]$ and $[0,1 / 3)$.

Proof of Lemma 12. Suppose that the trajectory touches the side $u_{i}=0$ or $u_{i}=1$ of $\partial N$ for some $i \in\{1, \ldots, n\}$. So that it stays in $N$ we must have $z_{i}=0$ at this point. Thus, we must consider these parts of $\partial N$, where $z_{i}=0$ for some $i$. So, suppose that a trajectory touches the side $z_{i}=0$ for $\xi=\xi_{0}$. As it does not leave the set $N$ we must also have $z_{i}^{\prime}\left(\xi_{0}\right)=0$ and $z^{\prime \prime}\left(\xi_{0}\right) \geq 0$. Hence $G_{\lambda i}\left(u\left(\xi_{0}\right)\right)=0$. However, differentiating the $i$-th equation we obtain the equation $a_{\lambda i}\left(u\left(\xi_{0}\right), z_{i}\left(\xi_{0}\right)\right) z_{i}^{\prime \prime}\left(\xi_{0}\right)+\sum_{j \neq i} G_{\lambda i, j}\left(u\left(\xi_{0}\right)\right) z_{j}\left(\xi_{0}\right)=0$, from which we infer, due to the monotonicity conditions, that $z_{i}^{\prime \prime}\left(\xi_{0}\right)<0$ unless $z_{j}\left(\xi_{0}\right)=0$ for all $j \in\{1, \ldots, n\}$. But then repeating the above arguments for each $j \neq i$ we conclude that $\left(u\left(\xi_{0}\right), z\left(\xi_{0}\right)\right)$ is a singular point.

According to the definition of $G_{\lambda}$ the similar lemma holds for $\lambda \in[1 / 3,2 / 3]$, but for $u$ lying inside the balls

$$
b(\mathbf{0}, \delta)=\{u:|u| \leq \delta\} \quad \text { and } \quad b(\mathbf{1}, \delta)=\{u:|\mathbf{1}-u| \leq \delta\} .
$$


Lemma 13. For $\lambda \in[1 / 3,2 / 3]$ and $q \in[-Q, Q]$

$I(N) \cap[\partial N \cap\{(u, z): u \in \operatorname{int}(b(\mathbf{0}, \delta)) \cup \operatorname{int}(b(\mathbf{1}, \delta))\}]=\{(u, \mathbf{0}), u=\mathbf{0}$ or $u=\mathbf{1}\}$.

The unstable singular points $\left(E_{k}, \mathbf{0}\right), k \in\{1, \ldots, K\}$ for $\lambda \in(2 / 3,1]$ and the unstable singular point $(E, \mathbf{0})$ for $\lambda \in[0,1 / 3)$ have the following crucial property given in the following lemma.

Lemma 14. For $\lambda \in(2 / 3,1]$ and $q \in[-Q, Q]$ there cannot exist simultaneously two solutions $\underline{u}, \bar{u}$ to system (10) satisfying the following conditions:

(1) $\left(\underline{u}(\xi), \underline{u}^{\prime}(\xi)\right) \rightarrow\left(E_{k}, \mathbf{0}\right)$ as $\xi \rightarrow \infty$, with $\underline{u}^{\prime}(\xi)>\mathbf{0}$,

(2) $\left(\bar{u}(\xi), \bar{u}^{\prime}(\xi)\right) \rightarrow\left(E_{k}, \mathbf{0}\right)$ as $\xi \rightarrow-\infty$, with $\bar{u}^{\prime}(\xi)>\mathbf{0}$,

for all $k=1, \ldots, K$.

For $\lambda \in[0,1 / 3)$ and $q \in[-Q, Q]$, there cannot exist simultaneously two solutions $\underline{u}, \bar{u}$ to system (10) such that:

(3) $\left(\underline{u}(\xi), \underline{u}^{\prime}(\xi)\right) \rightarrow(E, \mathbf{0})$ as $\xi \rightarrow \infty$, with $\underline{u}^{\prime}(\xi)>\mathbf{0}$,

(4) $\left(\bar{u}(\xi), \bar{u}^{\prime}(\xi)\right) \rightarrow(E, \mathbf{0})$ as $\xi \rightarrow-\infty$, with $\bar{u}^{\prime}(\xi)>\mathbf{0}$.

The following lemmas are of basic importance in the proof of Lemma 14.

Lemma 15 (see [18, Lemma 2.4, p. 161]). Let $\mathcal{A}$ be a constant diagonal matrix with positive entries. Let $F: \mathbb{R}^{n} \rightarrow \mathbb{R}^{n}$ be such that $F\left(u_{0}\right)=\mathbf{0}$ and $D F\left(u_{0}\right)$ has all of its off-diagonal elements positive. If there exists a solution $u(\xi)$ to the system

$$
\mathcal{A} u^{\prime \prime}+c u^{\prime}+F(u)=\mathbf{0}
$$

tending to $u_{0}$ as $\xi \rightarrow \infty(-\infty)$ and such that $u(\xi)<u_{0}\left(u(\xi)>u_{0}\right)$ for all suffciently large $\xi(-\xi)$, then there exist a number $\kappa \leq 0(\kappa \geq 0)$ and a positive vector $P$, such that

$$
\left[\mathcal{A} \kappa^{2}+c \kappa+D F\left(u_{0}\right)\right] P=\mathbf{0} .
$$

Lemma 16 (cf. Lemma 3.7 in [5]). Let $\mathcal{A}, C, B$ be $n \times n$ matrices with $B_{i j}>0$ if $j \neq i, \mathcal{A}, C$ diagonal with $\mathcal{A}$ positive-definite. Then the PerronFrobenius eigenvalue $\mu_{P F}\left(\eta^{2} \mathcal{A}+\eta C+B\right)$ is a strictly convex function of $\eta \in \mathbb{R}^{1}$. To be more precise, for $\eta_{1}, \eta_{2} \in \mathbb{R}^{1}, \eta_{1} \neq \eta_{2}$ and $0<t<1$, we have:

$$
\begin{aligned}
\mu_{P F}\left\{\left(t \eta_{1}+(1-t) \eta_{2}\right)^{2} \mathcal{A}+\left(t \eta_{1}+(1-t) \eta_{2}\right) C+B\right\} \\
\leq t \mu_{P F}\left\{\eta_{1}^{2} \mathcal{A}+\eta_{1} C+B\right\}+(1-t) \mu_{P F}\left(\eta_{2}^{2} \mathcal{A}+\eta_{2} C+B\right\} .
\end{aligned}
$$

Proof. The proof of this lemma is the same as the proof of Lemma 3.7 in [5]. According to the result of Cohen (see [5, Theorem 3.6]) for any matrix $\mathcal{M}$ with positive off-diagonal elements, any two real diagonal matrices $C_{1}, C_{2}$ and any $t \in(0,1)$ we have

$$
\mu_{P F}\left[t C_{1}+(1-t) C_{2}+\mathcal{M}\right] \leq t \mu_{P F}\left[C_{1}+\mathcal{M}\right]+(1-t) \mu_{P F}\left[C_{2}+\mathcal{M}\right] .
$$


For any $\eta_{1}, \eta_{2} \in \mathbb{R}^{1}$ with $\eta_{1} \neq \eta_{2}$ and $t \in(0,1)$ we have $\left(t \eta_{1}+(1-t) \eta_{2}\right)^{2}<$ $t \eta_{1}^{2}+(1-t) \eta_{2}^{2}$. So, using Lemma 3 , we obtain

$$
\begin{aligned}
\mu_{P F} & {\left[\left(t \eta_{1}+(1-t) \eta_{2}\right)^{2} \mathcal{A}+\left(t \eta_{1}+(1-t) \eta_{2}\right) C+B\right] } \\
& <\mu_{P F}\left[t\left(\eta_{1}^{2} \mathcal{A}+\eta_{1} C\right)+(1-t)\left(\eta_{2}^{2} \mathcal{A}+\eta_{2} C\right)+B\right] \\
& <t \mu_{P F}\left[\eta_{1}^{2} \mathcal{A}+\eta_{1} C+B\right]+(1-t) \mu_{P F}\left[\eta_{2}^{2} \mathcal{A}+\eta_{2} C+B\right] .
\end{aligned}
$$

The lemma is proved.

Proof of Lemma 14. The proof of this lemma follows by an appropiate modification of the proof of Lemma 2.4 p. 161 in [18] (see also [6]). Let us consider the case $\lambda \in(2 / 3,1]$. Suppose that both of the possibilities (1) and (2) occur. Then $u_{0}=E_{k}$. We will show that in each case the lemma similar to Lemma 15 holds. This will lead to a contradiction. Suppose that point (2) takes place. Let $H(\tau, \lambda)$ denote the matrix obtained from $D G_{\lambda}\left(u_{0}\right)$ by subtracting a sufficiently small number $\tau>0$ from its elements. We have

$$
\begin{aligned}
G_{\lambda}(u) & =D G_{\lambda}\left(u_{0}\right)\left(u-u_{0}\right)+O\left(\left(u-u_{0}\right)^{2}\right) \\
& =H(\tau, \lambda)\left(u-u_{0}\right)+\left\{D G_{\lambda}\left(u_{0}\right)-H(\tau, \lambda)\left(u-u_{0}\right)\right\}+O\left(\left(u-u_{0}\right)^{2}\right) \\
& =H(\tau, \lambda)\left(u-u_{0}\right)+\tau \widetilde{\mathbf{I}}\left(u-u_{0}\right)+O\left(\left(u-u_{0}\right)^{2}\right)
\end{aligned}
$$

where $\widetilde{\mathbf{I}}$ denotes the matrix with all of its entries equal to 1 .

First, we will prove that for all $i$ and all $(-\xi)$ sufficiently large we have $\bar{u}_{i}^{\prime}(\xi) \leq C\left|\bar{u}(\xi)-u_{0}\right|$. First, let us assume that $\bar{u}^{\prime}(\xi)$ tends to zero monotonically for all $\xi<\widetilde{\xi}$ as $\xi$ decreases. By multiplying the $i$-th equation by $\bar{u}_{i}^{\prime}(\xi)$, integrating from $(-\infty)$ to $\xi<\widetilde{\xi}$, and using the mean value theorem we obtain the inequality

$$
\begin{aligned}
& \left(\bar{u}_{i}^{\prime}(\xi)\right)^{2} \leq\left\{\left|J_{\lambda i}\left(q, u_{0}, \mathbf{0}\right)+o_{1}(1)\right| \bar{u}_{i}^{\prime}\left(\xi_{1}\right)\left(\bar{u}_{i}(\xi)-u_{0 i}\right)\right. \\
& \left.\quad+\left|D G_{\lambda i}\left(\bar{u}\left(\xi_{2}\right)\right)\left(\bar{u}\left(\xi_{3}\right)-u_{0}\right)\left(1+o_{2}(1)\right)\right|\left(\bar{u}_{i}(\xi)-u_{0 i}\right)\right\}\left(\frac{1}{2} a_{\lambda i}\left(u_{0}, \mathbf{0}\right)\right)^{-1},
\end{aligned}
$$

where $o_{1}, o_{2}$ tend to zero as $\xi \rightarrow-\infty$ and $\xi_{1}, \xi_{2}, \xi_{3} \in(-\infty, \xi)$. From this we obtain the inequality

$$
\left(\bar{u}^{\prime}(\xi)\right)^{2} \leq C_{1} \bar{u}^{\prime}(\xi)\left|\bar{u}(\xi)-u_{0}\right|+C_{2}\left|\bar{u}(\xi)-u_{0}\right|^{2}
$$

for some finite positive constants $C_{1}, C_{2}$. It is obvious that the last inequality can be fulfilled only if $\bar{u}^{\prime}(\xi) \leq C\left|\bar{u}(\xi)-u_{0}\right|$ with the constant $C$ independent of $\xi$.

Now, suppose that $\bar{u}_{i}^{\prime}(\xi)$ does not tend to 0 monotonically and suppose that the inequality $\bar{u}_{i}^{\prime}(\xi) \leq C\left|\bar{u}(\xi)-u_{0}\right|$ is not true. Then there would exist a sequence $\left\{\xi_{k}\right\}_{1}^{\infty}$ diverging to $(-\infty)$ such that $\bar{u}_{i}^{\prime}\left(\xi_{k}\right) \geq k\left|\bar{u}(\xi)-u_{0}\right|$ for any natural $k \geq 1$. Let us replace this sequence by the sequence $\left\{\widetilde{\xi}_{k}\right\}_{1}^{\infty}$, where $\widetilde{\xi}_{k}=\xi_{k}$, if $\bar{u}_{i}^{\prime}(\xi) \leq$ $\bar{u}_{i}^{\prime}\left(\xi_{k}\right)$ for all $\xi \leq \xi_{k}$, or, if it is not true, $\widetilde{\xi}_{k}<\xi_{k}$ is the first nearest point, where $\bar{u}_{i}^{\prime}$ attains the supremum on the set $\left(-\infty, \xi_{k}\right)$. Moreover, out of this subsequence 
we may choose a subsequence, which will be denoted in the same way, such that $\bar{u}_{i}^{\prime}\left(\widetilde{\xi}_{k}\right)<\bar{u}_{i}^{\prime}\left(\widetilde{\xi}_{k-1}\right)$ and

$$
\bar{u}_{i}^{\prime}(\xi) \leq \bar{u}_{i}^{\prime}\left(\widetilde{\xi}_{k}\right) \text { for } \xi \leq \widetilde{\xi}_{k}
$$

According to the definition of the sequence $\left\{\widetilde{\xi}_{k}\right\}_{1}^{\infty}$ we would have

$$
\bar{u}_{i}^{\prime}\left(\widetilde{\xi}_{k}\right)>C_{k}\left|\bar{u}_{i}\left(\widetilde{\xi}_{k}\right)-u_{0}\right|
$$

with $C_{k} \rightarrow \infty$ as $k \rightarrow \infty$. However, carrying out the same integration as in the monotonic case for $\xi$ replaced by $\xi_{k}$ (and taking advantage of (26)) we can prove that $\bar{u}_{i}^{\prime}\left(\xi_{k}\right) \leq C\left|\bar{u}\left(\xi_{k}\right)-u_{0}\right|$ with $C$ independent of $k$. This contradicition proves our claim. Let

$$
\begin{aligned}
& a_{\lambda}\left(u_{0}, \mathbf{0}\right)=\operatorname{diag}\left(a_{\lambda 1}\left(u_{0}, 0\right), \ldots, a_{\lambda n}\left(u_{0}, 0\right)\right), \\
& c_{\lambda}\left(u_{0}, \mathbf{0}\right)=\operatorname{diag}\left(c_{\lambda 1}\left(u_{0}, \mathbf{0}\right), \ldots, c_{\lambda n}\left(u_{0}, \mathbf{0}\right)\right), \\
& M\left(u_{0}, \mathbf{0}\right)=\operatorname{diag}\left(M_{1}\left(u_{0}, \mathbf{0}\right), \ldots, M_{n}\left(u_{0}, \mathbf{0}\right)\right) .
\end{aligned}
$$

Near the point $\left(u_{0}, \mathbf{0}\right)$ system $(10)$ can be written in the following way:

$$
\begin{aligned}
& a_{\lambda}\left(u_{0}, \mathbf{0}\right) u^{\prime \prime}(\xi)-q c_{\lambda}\left(u_{0}, \mathbf{0}\right) u^{\prime}(\xi)+\psi(\lambda) M\left(u_{0}, \mathbf{0}\right) u^{\prime}(\xi) \\
&+H(\tau, \lambda)\left(u(\xi)-u_{0}\right)+\widetilde{H}(\tau, \lambda, \xi)=\mathbf{0},
\end{aligned}
$$

where by the use of Lagrange mean-value theorem

$$
\begin{aligned}
\widetilde{H}_{i}(\tau, \lambda, \xi)= & G_{\lambda i}(u(\xi))-\sum_{j=1}^{n} H_{i j}(\tau, \lambda)\left(u_{j}(\xi)-u_{0 j}\right)+\tau \sum_{j=1}^{n}\left(u_{j}(\xi)-u_{0 j}\right) \\
& +\psi(\lambda) u_{i}^{\prime \prime}(\xi)\left[\sum_{j=1}^{n}\left(u_{j}(\xi)-u_{0 j}\right) A_{1 i j}(\xi)+u_{i}^{\prime}(\xi) A_{2 i}(\xi)\right] \\
& +\psi(\lambda) u_{i}^{\prime}(\xi)\left[\sum_{j=1}^{n}\left(u_{j}(\xi)-u_{0 j}\right) A_{3 i j}(\xi)+\sum_{j=1}^{n} u_{j}^{\prime}(\xi) A_{4 i j}(\xi)\right],
\end{aligned}
$$

where $A_{1 i j}, A_{2 i}, A_{3 i j}, A_{4 i j}$ are bounded in $C^{1}$-norm. The fact that $\bar{u}_{j}(\xi) \rightarrow$ $u_{0 j}, \bar{u}_{j}^{\prime}(\xi) \rightarrow 0, \bar{u}_{j}^{\prime \prime}(\xi) \rightarrow 0$ implies that for any $\tau>0$ sufficiently small there exists $\xi^{*}=\xi^{*}(\tau)$ with $\left(-\xi^{*}\right)$ sufficiently large such that for all $\xi \leq \xi^{*}$ the function $\widetilde{H}(\tau, \lambda, \xi)$ is positive. Hence $\bar{u}(\xi)$ is a supersolution to the boundary value problem:

$$
\begin{gathered}
-U_{, t}+a_{\lambda}\left(u_{0}, \mathbf{0}\right) U^{\prime \prime}-q c_{\lambda}\left(u_{0}, \mathbf{0}\right) U^{\prime}+\psi(\lambda) M\left(u_{0}, \mathbf{0}\right) U^{\prime}+H(\tau, \lambda)\left(U-u_{0}\right)=\mathbf{0}, \\
U\left(t, \xi^{*}\right)=u\left(\xi^{*}\right), \quad U(0, \xi)=u(\xi) .
\end{gathered}
$$

One can show existence of a time independent function $v(\xi)$ such that $v(\xi)>u_{0}$ for $\xi<\xi^{*}, v(\xi) \rightarrow u_{0}$ as $\xi \rightarrow-\infty$ satisfying the last system of equations 
(see [18]). From Lemma 15 it follows that there exists a number $\kappa_{\tau} \geq 0$ and a positive vector $P_{\tau},\left|P_{\tau}\right|=1$, such that

$$
\left\{a_{\lambda}\left(u_{0}, \mathbf{0}\right) \kappa_{\tau}^{2}-\left[q c_{\lambda}\left(u_{0}, \mathbf{0}\right)+\psi(\lambda) M\left(u_{0}, \mathbf{0}\right)\right] \kappa_{\tau}+H(\tau, \lambda)\right\} P_{\tau}=\mathbf{0},
$$

Letting $\tau \rightarrow 0$ we infer that there exists a number $\kappa \geq 0$ and a vector $P \geq \mathbf{0}$ satisfying the relation

(28) $T(\kappa, q) P=\left\{a_{\lambda}\left(u_{0}, \mathbf{0}\right) \kappa^{2}-\left[q c_{\lambda}\left(u_{0}, \mathbf{0}\right)-\psi(\lambda) M\left(u_{0}, \mathbf{0}\right)\right] \kappa+D G_{\lambda}\left(u_{0}\right)\right\} P=\mathbf{0}$.

One can show (see the proof of Lemma 2.4 in [18]) that $P>\mathbf{0}$. In the same way, by considering the solution satisfying point 1 . we can arrive at the conclusion that there exists a number $\widetilde{\kappa} \leq 0$ and a vector $\widetilde{P}>\mathbf{0},|\widetilde{P}|=1$, satisfying relation

(29) $T(\widetilde{\kappa}, q) \widetilde{P}=\left\{a_{\lambda}\left(u_{0}, \mathbf{0}\right) \widetilde{\kappa}^{2}-\left[q c_{\lambda}\left(u_{0}, \mathbf{0}\right)-\psi(\lambda) M\left(u_{0}, \mathbf{0}\right)\right] \widetilde{\kappa}+D G_{\lambda}\left(u_{0}\right)\right\} \widetilde{P}=\mathbf{0}$.

As, according to Assumption 2, $\mu_{P F}\left(D G_{\lambda}\left(u_{0}\right)\right)>0$, then $\kappa>0$ and $\widetilde{\kappa}<0$. Let $\eta_{1}=\kappa$ and $\eta_{2}=\widetilde{\kappa}$. We will prove that for fixed $q$ both of the relations (28), (29) cannot be satisfied. If both of these relations were satified, then for $\eta=\eta_{1}$ and $\eta=\eta_{2}$ the Perron-Frobenius eigenvalues of the matrix

$$
T(\eta, q)=a_{\lambda}\left(u_{0}, \mathbf{0}\right) \eta^{2}-\left[q c_{\lambda}\left(u_{0}, \mathbf{0}\right)-\psi(\lambda) M\left(u_{0}, \mathbf{0}\right)\right] \eta+D G_{\lambda}\left(u_{0}\right)
$$

would be equal to 0 . However, we can find $t \in(0,1)$ such that $t \eta_{1}+(1-t) \eta_{2}=0$. Hence according to Lemma 16 we would have

$$
\begin{aligned}
0< & \mu_{P F}\left(D G_{\lambda}\left(u_{0}\right)\right) \\
\leq & t\left\{a_{\lambda}\left(u_{0}, \mathbf{0}\right) \eta_{1}^{2}-\left[q c_{\lambda}\left(u_{0}, \mathbf{0}\right)-\psi(\lambda) M\left(u_{0}, \mathbf{0}\right)\right] \eta_{1}+D G_{\lambda}\left(u_{0}\right)\right\} \\
& +(1-t)\left\{a_{\lambda}\left(u_{0}, \mathbf{0}\right) \eta_{2}^{2}-\left[q c_{\lambda}\left(u_{0}, \mathbf{0}\right)-\psi(\lambda) M\left(u_{0}, \mathbf{0}\right)\right] \eta_{2}+D G_{\lambda}\left(u_{0}\right)\right\}=0 .
\end{aligned}
$$

This leads to a contradiction.

Similarly, we prove that points (3) and (4) cannot take place simultaneously. Thus Lemma 14 is proved.

The next lemma claims that the intermediate singular points are the maximal invariant sets in some suffciently small neighbourhoods of them. We say that they are invariant relative to $I(N)$ (see Chapter D, p. 334 in [4]).

LEMma 17. For every $\lambda \in[0,1 / 3)$ there exists a sufficiently small open neighbourhood $V_{E}(\lambda)$ of $(E, 0)$ such that $(E, 0)$ is the maximal invariant set in $V_{E}(\lambda) \cap N$. For every $\lambda \in(2 / 3,1]$ and every $k=1, \ldots, K$ there exists a sufficiently small open neighbourhood $V_{E_{k}}(\lambda)$ of the singular point $\left(E_{k}, 0\right)$ such that $\left(E_{k}, 0\right)$ is the maximal invariant set in $V_{E_{k}}(\lambda) \cap N$.

Proof. Suppose that the first claim is not true and that there exists a trajectory $(u(\xi), z(\xi))$ contained completely in $V_{E}(\lambda) \cap N$. According to Lemma 14 this trajectory cannot tend to the point $(E, 0)$ both for $\xi \rightarrow \infty$ and $\xi \rightarrow-\infty$. 
Let us suppose that it tends to this point only for $\xi \rightarrow-\infty(\xi \rightarrow \infty)$. As there is no other singular point in some vicinity of $(E, 0)$, then due to Lemma 12 $z^{\prime}(\xi)>\varepsilon_{z}>0$ for all $\xi>0$ (or $\xi<0$ ). Hence for $\xi$ (or $-\xi$ ) suffciently large this trajectory must leave the set $V_{E}(\lambda) \cap N$. Similarly we can prove that for every $\lambda \in[0,1 / 3)$ and every $k=1, \ldots, K$ there exists a sufficiently small open neighbourhood (in $\left.\mathbb{R}^{2 n}\right) V_{E_{k}}(\lambda)$ of the singular point $\left(E_{k}, 0\right)$ such that $\left(E_{k}, 0\right)$ is the maximal invariant set in $V_{E_{k}}(\lambda) \cap N$.

Definition 4. For $\lambda \in[0,1 / 3)$ and $q \in[-Q, Q]$ let $A_{+}^{*}(\lambda, q)\left(A_{-}^{*}(\lambda, q)\right)$ denote the set of points lying on the trajectories of solutions $(u(\xi), z(\xi))$ to system (15) belonging to $I(N)$ such that $\lim _{\xi \rightarrow \infty} u(\xi)=E,\left(\lim _{\xi \rightarrow-\infty} u(\xi)=\right.$ $E)$, and $\lim _{\xi \rightarrow \infty} z(\xi)=0\left(\lim _{\xi \rightarrow-\infty} z(\xi)=0\right)$. For $\lambda \in(2 / 3,1]$ and $q \in$ $[-Q, Q]$ let $A_{k+}(\lambda, q)\left(A_{k-}(\lambda, q)\right)$ denote the set of points lying on the trajectories of solutions $(u(\xi), z(\xi))$ belonging to $I(N)$ such that $\lim _{\xi \rightarrow \infty} u(\xi)=E_{k}$ $\left(\lim _{\xi \rightarrow-\infty} u(\xi)=E_{k}\right)$, and $\lim _{\xi \rightarrow \infty} z(\xi)=0\left(\lim _{\xi \rightarrow-\infty} z(\xi)=0\right)$.

From Lemma 14 one can easily derive the important properties of the sets defined in Definition 4.

Lemma 18. For each $\lambda \in[0,1 / 3), q \in[-Q, Q]$ at least one of the sets $A_{+}^{*}(\lambda, q), A_{-}^{*}(\lambda, q)$ must be empty. As well for all $\lambda \in(2 / 3,1], q \in[-Q, Q]$ and all $k \in\{1, \ldots, K\}$, at least one of the sets $A_{k+}(\lambda, q), A_{k-}(\lambda, q)$ must be empty.

Using Lemma 14 and its proof one can also give a more precise characterization of these sets.

Lemma 19. For $\lambda \in[0,1 / 3), A_{+}^{*}(\lambda, q)=\emptyset$ if $q \geq 0$ and $A_{-}^{*}(\lambda, q)=\emptyset$ if $q \leq 0$. For $\lambda \in(2 / 3,3 / 4], k \in K, A_{k+}(\lambda, q)=\emptyset$ for $q \geq 0$ and $A_{k-}(\lambda, q)=\emptyset$ for $q \leq 0$. For $\lambda \in(3 / 4,1], k \in K$, there exist numbers $q_{* k}(\lambda)$ and $q_{k}^{*}(\lambda) \geq q_{* k}(\lambda)$ such that $A_{k+}(\lambda, q)=\emptyset$ for $q \geq q_{k}^{*}(\lambda)$ and $A_{k-}(\lambda, q)=\emptyset$ for $q \leq q_{* k}(\lambda)$. Moreover, the sets $\left\{q \in \mathbb{R}^{1}: A_{k+}(\lambda, q)=\emptyset\right\}$ and $\left\{q \in \mathbb{R}^{1}: A_{k-}(\lambda, q)=\emptyset\right\}$ are simply connected.

Proof. For $\lambda \leq 3 / 4$ we have $\psi(\lambda) \equiv 0$, hence the matrix $T(\eta, q)$ given by (30) takes the form

$$
\eta^{2} I-\eta q u^{\prime} \eta I+D G_{\lambda}\left(u_{0}\right) .
$$

If $q \leq 0$ and $\eta \geq 0$ then the maximal eigenvalue of this matrix is positive, because it is positive for $\eta=0$ (cf. Lemma 3 and the proof of Lemma 4). The same arguments work if $q \geq 0$ and $\eta \leq 0$. Hence the first two claims of the lemma are true. If $\lambda \in(3 / 4,1]$ then there exists $q_{k}^{*}(\lambda)$ so large that $\left[-q c_{\lambda}\left(u_{0}, \mathbf{0}\right)+\right.$ $\left.\psi(\lambda) M\left(u_{0}, \mathbf{0}\right)\right] \leq 0$ for $q \geq q_{k}^{*}(\lambda)$ and $q_{* k}(\lambda)$ sufficiently close to $(-\infty)$ such that $\left(-q c_{\lambda}\left(u_{0}, \mathbf{0}\right)+\psi(\lambda) M\left(u_{0}, \mathbf{0}\right)\right] \geq 0$ for $q \leq q_{* k}(\lambda)$. Hence as above we can prove the third statement of the lemma. Now, let $q_{1} \in\left\{q \in \mathbb{R}^{1}: A_{k+}(\lambda, q)=\emptyset\right\}$ and suppose that there exists $q_{2}>q_{1}$ such that $\mu_{P F}\left(T\left(\eta_{2}, q_{2}\right)\right)=0$ for some 
$\eta_{2}<0$. This would mean that the matrix $T\left(\eta_{2}, q_{1}\right)$ has its diagonal elements strictly smaller than those of the matrix $T\left(\eta_{2}, q_{2}\right)$, hence $\mu_{P F}\left(T\left(\eta_{2}, q_{1}\right)\right)<0$. As $\mu_{P F}\left(T\left(0, q_{1}\right)\right)>0$ then by continuity of $\mu_{P F}\left(T\left(\eta, q_{1}\right)\right)$ with respect to $\eta$ it would be possible to find $\eta_{1}, 0>\eta_{1}>\eta_{2}$, such that $\mu_{P F}\left(T\left(q_{1}, \eta_{1}\right)\right)=0$. This is a contradiction. In the similar way we prove that the set $\left\{q \in \mathbb{R}^{1}: A_{k-}(\lambda, q)=\emptyset\right\}$ is simply connected.

Now, we will analyze the set $I(N) \cap \partial N$ for $\lambda \in[1 / 3,2 / 3], q \in[-Q, Q]$. Note that for these values of $\lambda$ we are dealing with the system:

$$
u_{i}^{\prime \prime}-q u_{i}^{\prime}+G_{\lambda i}(u)=0,
$$

$i=1, \ldots, n$. Moreover, $G_{\lambda i}(u) \equiv 0$ for $u \notin \operatorname{int}(b(\mathbf{0}, \delta)) \cup \operatorname{int}(b(\mathbf{1}, \delta))$.

Definition 5. Let $\mathcal{P}=\{(u, z):(u, z) \in N, z=\mathbf{0}, u \notin \operatorname{int}(b(\mathbf{0}, \delta)) \cup$ $\operatorname{int}(b(\mathbf{1}, \delta))\}$. For $\lambda \in[1 / 3,2 / 3]$ and $q \in[-Q, Q]$ let $A_{+}(\lambda, q)\left(A_{-}(\lambda, q)\right)$ denote the set of points lying on the trajectories of solutions $(u(\xi), z(\xi))$ belonging to $I(N)$ such that $\lim _{\xi \rightarrow \infty}(u(\xi), z(\xi)) \in \mathcal{P}\left(\lim _{\xi \rightarrow-\infty}(u(\xi), z(\xi)) \in \mathcal{P}\right)$.

(Note that for every trajectory from $N$ the $\operatorname{limits}_{\lim _{\xi \rightarrow \pm \infty}}(u(\xi), z(\xi))$ are well defined.) Our aim is to excise a sufficiently small neighbourhood of the set $\mathcal{P}$ out of $N^{*}$ so that it becomes an isolating neighbourhood. However, before doing that, we must check whether the sets $A_{+}(\lambda, q)$ and $A_{-}(\lambda, q)$ have the proper structure. We want to use the Lemma in Chapter D, p. 334 in [4]. For the reader's convenience we cite it here.

Let $Z$ be a compact invariant set and let $\widehat{Z}$ be a subset of $Z$. Then $\widehat{Z}$ is called an isolated invariant set relative to $Z$ if there is a compact neighbourhood $\widehat{N}$ of $\widehat{Z}$ in $Z$ such that $\widehat{Z}=I(\widehat{Z})$. If this is the case let $A^{+}=A^{+}(\widehat{Z}, Z)$ (respectively, $\left.A^{-}(\widehat{Z}, Z)\right)$ be the set of points on solutions in $Z \backslash \widehat{Z}$ that tend to $\widehat{Z}$ in forward time (respectively, backward time).

Lemma 20 ([4, p. 334]). Suppose that $N$ is compact. Let $Z=I(N)$ and let $\widehat{Z}$ be isolated relative to $Z$. Suppose that either $A^{+}=A^{+}(\widehat{Z}, Z)$ or $A^{-}(\widehat{Z}, Z)$ is empty. Then, for all small enough neighbourhoods $U$ of $\widehat{Z}$,

$$
I(N \backslash U) \cap \partial(N \backslash U)=I(N) \cap \partial N \backslash\left(A^{+} \cup A^{-} \cup \widehat{Z}\right) .
$$

For the reader's convenience we give the proof of Lemma 20 in Appendix.

The analysis of the sets $A_{+}(\lambda, q)$ and $A_{-}(\lambda, q)$ will be divided into three cases: $q=0, q>0$ and $q<0$.

Case I. $q=0$.

Lemma 21. For $\lambda \in[1 / 3,2 / 3]$ and $q=0$ both $A_{+}(\lambda, q)$ and $A_{-}(\lambda, q)$ are empty and $I(N) \cap \partial N^{*}=\mathcal{P}$. Moreover, $\mathcal{P}$ is the maximal invariant set in the closure of a sufficiently small neighbourhood of $\mathcal{P}$ contained in $N$. 
Proof. Suppose that a trajectory $(u(\xi), z(\xi))$ touches the part of the boundary given by the relation $u_{i}=0$ or $u_{i}=1$ at some point $(U, Z)$. So that this trajectory does not leave the set $N$ we must have $Z_{i}=0$ at this point. Thus the problem is reduced to the analysis of these parts of the boundary at which $z_{i}=0$.

(a) Suppose that $U$ lies outside the open balls $b(\mathbf{0}, \delta)=\{u:|u| \leq \delta\}$ and $b(\mathbf{1}, \delta)=\{u:|\mathbf{1}-u| \leq \delta\}$. Suppose first that $Z_{j}=0$ for all $j \in\{1, \ldots, n\}$. Then $(U, Z)=(U, \mathbf{0})$ is a singular point. Obviously this singular point is not isolated, because in every arbitrarily small neighbourhood of it there are other singular points belonging to $\mathcal{P}$. But no other trajectory passes through it nor tends to it as $\xi \rightarrow \pm \infty$. (To see this, note that sufficiently close to this point system (10) becomes simply $z_{j}^{\prime}=0$.) Thus suppose that $Z_{j}>0$ for some $j$ and that $(U, Z)=\left(u\left(\xi_{0}\right), z\left(\xi_{0}\right)\right)$. Then, as $z_{i}(\xi)=$ const as long as $u(\xi)$ stays outside $b(\mathbf{0}, \delta)$ and $b(\mathbf{1}, \delta)$ the projection of this trajectory onto $u$-space will be contained in the plane $u_{i}=$ const $=u_{i}\left(\xi_{0}\right)$. It will thus have the form $u_{j}(\xi)=z_{j}\left(\xi_{0}\right)\left(\xi-\xi_{0}\right)$. But, any trajectory of this form will cross the boundary of the set $[0,1]^{n}$ for $\left(\xi-\xi_{0}\right)$ or $\left(-\left(\xi-\xi_{0}\right)\right)$ sufficiently large. (To see this, let us note that, as $\delta<1 / 2$, at most for one of the balls $b(\mathbf{0}, \delta)$ and $b(\mathbf{1}, \delta)$ the intersection with the plane $u_{i}=u_{i}\left(\xi_{0}\right)$ is nonempty independently of $u_{i}\left(\xi_{0}\right)$.) Hence the point $(U, Z)$ with $Z_{j}>0$ for at least one $j$ cannot belong to $I(N)$.

(b) Suppose that $U=u\left(\xi_{0}\right)$ lies at the boundary of $b(\mathbf{0}, \delta)$. If we assume that $z_{j}\left(\xi_{0}\right)>0$ for at least one $j$, then, by repeating the arguments from point (a), we note that the trajectory through the point $(U, Z)$ will leave the set $N$, so this point will not belong to $I(N)$. So, let us assume that $Z_{j}=0$ for all $j$. Suppose that there exists a trajectory belonging to $I(N)$, which tends the point $(U, \mathbf{0})$ as $\xi \rightarrow \infty$. It follows that $z_{j}(\xi) \geq 0, z_{j}(\xi) \not \equiv 0, \xi \in \mathbb{R}^{1}$ for at least one $j$. Hence $u(\xi)$ lies inside the ball $b(\mathbf{0}, \delta)$ for all $\xi \in \mathbb{R}^{1}$. From Lemma 13 we conclude that if the considered trajectory touched that part of the boundary at which $Z_{j}=0$ for some $j \in\{1, \ldots, n\}$ and $u \in \operatorname{int}(b(\mathbf{0}, \delta)$, then it would not belong to $I\left(N^{*}\right)$. (Remind that there is no singular point with $|u|<\delta$ except for $(\mathbf{0}, \mathbf{0})$.) In consequence $z_{j}(\xi)>0$ for all $\xi \in \mathbb{R}^{1}$ for all $j$. Now, let us note for all $j \in\{1, \ldots, n\}$ there exists a sequence $\left\{\xi_{J}^{j}\right\}_{J=1}^{\infty}$ such that $\xi_{J}^{j} \rightarrow \infty$ as $J \rightarrow \infty$ and $z_{j}^{\prime}\left(\xi_{J}^{j}\right)<0$. (Otherwise, $z_{j}(\xi)$ could not tend to 0 , because $z_{j}(\xi)>0$ and its derivative would be nonnegative for $\xi$ sufficiently large.) As $\left(\omega\left(u\left(\xi_{J}^{j}\right)\right)\right)^{-1} z_{j}^{\prime}\left(\xi_{J}^{j}\right)=-\left(\omega\left(u\left(\xi_{J}^{j}\right)\right)\right)^{-1} G_{\lambda j}\left(u\left(\xi_{J}^{j}\right)\right)$ and $u\left(\xi_{J}^{j}\right) \rightarrow U$ as $J \rightarrow \infty$, it follows, due to the positveness of $\omega(u)$ for $|u|<\delta$, that $(\omega(U))^{-1} G_{\lambda j}(U) \geq 0$. However, this situation is impossible if $\delta$ is chosen sufficiently small, according Lemma 8. Thus a trajectory with the above prescribed properties does not exist. Moreover, there does not exist an invariant trajectory contained in $N$ tending to $(U, Z)$ as $\xi \rightarrow-\infty$. If such a trajectory existed, then there would be $\xi_{0} \in(-\infty, 0)$ 
such that $u\left(\xi_{0}\right) \notin b(\mathbf{0}, \delta)$ and $z_{j}\left(\xi_{0}\right)>0$ at least for one $j$. (Otherwise it would be a singular point.) But, as $z_{j}^{\prime}(\xi)=0$ for all $\xi \in\left(-\infty, \xi_{0}\right]$, it would mean that $\lim _{\xi \rightarrow-\infty} z_{j}(\xi)>0$, which is a contradiction. The same arguments can be applied to $\partial b(\mathbf{1}, \delta)$ (with different direction of time).

This proves the first two statements of the lemma. The third statement follows from the arguments above. First, if $(u, z)$ is such that $u \notin b(\mathbf{0}, \delta) \cup b(\mathbf{1}, \delta)$ and $z \neq \mathbf{0}$, then by (a) $(u, z) \notin I(N)$. If $u$ lies at the boundary of these balls, then by (b) the same conclusion holds. If $u$ lies inside these balls and $z_{j}=0$ for some $j$, then by Lemmas 11 and 13 also $(u, z) \notin I(N)$. Finally suppose that $u \in b(\mathbf{0}, \delta)$ and $z_{j} \neq 0$, for all $j$. Then the trajectory through this point will leave any sufficiently small closed neighbourhood of $\mathcal{P}$ (in $N$ ) as $\xi \rightarrow-\infty$ unless $z_{k}(\xi)$ becomes equal to 0 for at least one $k$. But this situation is impossible according to Lemma 13. The same arguments hold, if $u \in b(\mathbf{1}, \delta)$. The lemma is proved. $\square$

Case II. $q>0$.

Lemma 22. For $\lambda \in[1 / 3,2 / 3]$ and $q>0$ the set $A_{+}(\lambda, q)$ is empty, whereas $\left[I(N) \cap \partial N^{*} \backslash \mathcal{P}\right] \subset A_{-}(\lambda, q)$. Moreover, $\mathcal{P}$ is the maximal invariant set in the closure of a sufficiently small neighbourhood of $\mathcal{P}$ contained in $N$.

Proof. Suppose that a trajectory $(u(\xi), z(\xi))$ touches at the point $(U, Z)$ the part of the boundary given by the relation $u_{i}=0$. So that this trajectory does not leave the set $N$ we must have $Z_{i}=0$. Thus the problem is reduced to the analysis of that part of the boundary at which $z_{i}=0$.

(a) Suppose that $U$ lies outside the closed balls $b(\mathbf{0}, \delta)$ and $b(\mathbf{1}, \delta)$. Suppose that $Z_{j}=0$ for all $j$. Then $(U, Z)=(U, \mathbf{0})$ is a singular point. As in the first case this singular point is not isolated, because in every arbitrarily small neighbourhood of it there are other singular points belonging to $\mathcal{P}$, But no other trajectory passes through it or tends to it as $\xi \rightarrow \infty$. (To see this, note that sufficiently close to this point system (10) becomes simply $z_{j}^{\prime}=q z_{j}, j \in\{1, \ldots, n\}$.) Now, suppose that some $Z_{j}$ are positive and that $(U, Z)=\left(u\left(\xi_{0}\right), z\left(\xi_{0}\right)\right)$. Then, for positive $\xi-\xi_{0}$ the projection of the trajectory starting from the considered point has the form

(31) $\left(u_{j}(\xi), z_{j}(\xi)\right)=\left(u_{j}\left(\xi_{0}\right)+z_{j}\left(\xi_{0}\right) q^{-1}\left\{\exp \left[q\left(\xi-\xi_{0}\right)\right]-1\right\}, z_{j}\left(\xi_{0}\right) \exp \left[q\left(\xi-\xi_{0}\right)\right]\right)$,

$j=1, \ldots, n$. The $u$ projection of this trajectory is a straight line starting from the point $u\left(\xi_{0}\right)$ and lying in the plane $u_{i}=$ const $=u_{i}\left(\xi_{0}\right)$ as long as $u(\xi) \notin b(\mathbf{0}, \delta) \cup b(\mathbf{1}, \delta)$. Thus, if $u_{i}\left(\xi_{0}\right) \leq 1-\delta$, then any forward trajectory (for $\left.\xi>\xi_{0}\right)$ will leave the set $N^{*}$. So, let us assume that $u_{i}\left(\xi_{0}\right)>1-\delta$. For $\xi>\xi_{0}$ sufficiently large the trajectory may get inside the ball $b(\mathbf{1}, \delta)$. As the value of $\xi-\xi_{0}$ decreases, it either leaves $N^{*}$ or tends, as $\xi \rightarrow-\infty$, to the singular point $(u, \mathbf{0}) \in \mathcal{P}$ where $u=\left(u_{1}\left(\xi_{0}\right)-z_{1}\left(\xi_{0}\right) q^{-1}, \ldots, u_{n}\left(\xi_{0}\right)-z_{n}\left(\xi_{0}\right) q^{-1}\right)$. (The last 
claim follows from the fact that $\delta \in(0,1 / 2)$.) However, it is obvious from (31) and from the fact that $z_{j}$ satisfies the equation $z_{j}^{\prime}=q z_{j}$ that for such a point there does not exist a trajectory in $N^{*}$ tending to it as $\xi \rightarrow \infty$.

(b) Let us consider the trajectories tending to the points of the form $(U, Z)=$ $(U, \mathbf{0}), U \in \partial b(\mathbf{0}, \delta)$, as $\xi \rightarrow \infty$. We may repeat the proof from the point $\mathrm{I}(\mathrm{b})$ to claim that such a trajectory cannot belong to $I(N)$. Finally, if $U \in \partial b(\mathbf{1}, \delta)$, then also does not exist a trajectory in $I(N)$ tending to the considered point as $\xi \rightarrow \infty$. (If such a trajectory existed then for at least one $j$ we would have $u_{j}(\xi) \notin b(\mathbf{0}, \delta) \cup b(\mathbf{1}, \delta)$ for all $\xi \geq \Xi, \Xi<\infty$, and $z_{j}(\cdot)$ would not be equal to 0 on this set. Then however $z_{j}^{\prime}(\xi)=q z_{j}(\xi) \geq 0$, so we could not have $\lim _{\xi \rightarrow \infty} z_{j}(\xi)=0$.)

The last claim of the lemma can be proved as in Lemma 21. The lemma is proved.

Case III. $q<0$. As in the case $q>0$ we may prove the following lemma.

Lemma 23. For $\lambda \in[1 / 3,2 / 3]$ and $q<0$ the set $A_{-}(\lambda, q)$ is empty, whereas $\left[I(N) \cap \partial N^{*} \backslash \mathcal{P}\right] \subset A_{+}(\lambda, q)$. Moreover, $\mathcal{P}$ is the maximal invariant set in the closure of a sufficiently small neighbourhood of $\mathcal{P}$ contained in $N$.

Now, we will modify the set $N^{*}$ continuously with respect to $\lambda$ in such a way that it becomes an isolating neighbourhood. First, using Lemmas 20, 12 and 18 we infer that for $\lambda \in[0,1 / 3)$ and all $q \in[-Q, Q]$ there exists $\gamma_{\lambda}>0$ such that

$$
N_{*}(\lambda)=N^{*} \backslash \mathcal{B}\left((E, 0), \gamma_{\lambda}\right),
$$

where $\mathcal{B}\left((E, 0), \gamma_{\lambda}\right)$ is an open ball with centre at $(E, 0)$ and radius $\gamma_{\lambda}$, is an isolating neighbourhood as

$$
\begin{aligned}
I\left(N_{*}(\lambda)\right) \cap \partial N_{*}(\lambda) & =I(N) \cap \partial N^{*} \backslash\left[(E, 0) \cup A_{+}^{*}(\lambda, q) \cup A_{-}^{*}(\lambda, q)\right] \\
& =I(N) \cap \partial N^{*} \backslash(E, 0)=\emptyset .
\end{aligned}
$$

Likewise, according to Lemmas 20,12 and 18 , for $\lambda \in(2 / 3,1]$ and all $q \in[-Q, Q]$ there exists $\gamma_{\lambda}>0$ such that

$$
N_{*}(\lambda)=N^{*} \backslash \bigcup_{k} \mathcal{B}\left(\left(E_{k}, 0\right), \gamma_{\lambda}\right)
$$

is an isolating neighbourhood as

$$
\begin{aligned}
I\left(N_{*}(\lambda)\right) \cap \partial N_{*}(\lambda) & =I(N) \cap \partial N^{*} \backslash\left[\bigcup_{k}\left(E_{k}, 0\right) \cup \bigcup_{k} A_{k+}(\lambda, q) \cup \bigcup_{k} A_{k-}(\lambda, q)\right] \\
& =I(N) \cap \partial N^{*} \backslash\left[\bigcup_{k}\left(E_{k}, 0\right)\right]=\emptyset .
\end{aligned}
$$


According to Lemmas 21-23 and Lemma 20 for all $\lambda \in[1 / 3,2 / 3]$ and all $q \in$ $[-Q, Q]$ there exists an open neighbourhood $V \subset \mathbb{R}^{2 n}$ of the set $\mathcal{P}$ such that

$$
N_{*}(\lambda)=N^{*} \backslash V
$$

is an isolating neighbourhood, i.e.

$$
I\left(N_{*}(\lambda)\right) \cap \partial N_{*}(\lambda)=I(N) \cap \partial N^{*} \backslash\left(\mathcal{P} \cup A_{+}(\lambda, q) \cup A_{-}(\lambda, q)\right)=\emptyset .
$$

Due to the properties of isolating neighbourhoods $N^{*} \backslash V$ is an isolating neighbourhood for all $\lambda \in[1 / 3-2 \Delta \lambda, 2 / 3+2 \Delta \lambda]$ for some $\Delta \lambda>0$ sufficiently small. Then we can choose $\gamma>0$ so small that $\mathcal{B}((E, 0), \gamma) \subset V$ for $\lambda \in$ $[1 / 3-2 \Delta \lambda, 1 / 3-\Delta \lambda], \bigcup_{k} \mathcal{B}\left(\left(E_{k}, 0\right), \gamma\right) \subset V$ for $\lambda \in[2 / 3+\Delta \lambda, 2 / 3+2 \Delta \lambda]$, and $\gamma \leq \gamma_{\lambda}$ for all $\lambda \in[0,1 / 3-\Delta \lambda] \cup[2 / 3+\Delta \lambda, 1]$. Hence we can construct a continuous in $\lambda \in[0,1]$ family of open sets $V(\lambda)$ such that $V(\lambda)=V$ for $\lambda \in[1 / 3-\Delta \lambda, 2 / 3+\Delta \lambda], V(\lambda)=\mathcal{B}((E, 0), \gamma), \lambda \in[0,1 / 3-2 \Delta \lambda], V(\lambda)=$ $\bigcup_{k} \mathcal{B}\left(\left(E_{k}, 0\right), \gamma\right), \lambda \in[2 / 3+2 \Delta \lambda, 1]$ and $V(\lambda) \subset V$ for $\lambda \in(1 / 3-2 \Delta \lambda, 1 / 3-$ $\Delta \lambda) \cup(2 / 3+\Delta, 2 / 3+2 \Delta)$. Consequently for all $\lambda \in[0,1], q \in[-Q, Q]$ the set

$$
N_{\lambda}=N^{*} \backslash V(\lambda)
$$

is an isolating neighbourhood.

\section{Use of the Conley connection index theory}

First, let us state an obvious lemma.

Lemma 24. There exists $\widetilde{\varepsilon}>0$ (sufficiently small) such that for all $\lambda \in[0,1]$ and all $q \in[-Q, Q]$ the closed balls $\mathcal{B}(A, \widetilde{\varepsilon})$ and $\mathcal{B}(A, \widetilde{\varepsilon})$ are isolating neighbourhoods. The maximal invariant sets in these balls are the singular points $(\mathbf{0}, \mathbf{0})$ and $(\mathbf{1}, \mathbf{0})$ respectively. The Conley index of these sets is equal to $\Sigma^{n}$.

Proof. According to Lemma 10 the sets $(\mathbf{0 , 0})$ and $(\mathbf{1}, \mathbf{0})$ are saddle points and the linearization matrix of system (15) at these points have exactly $n$ eigenvalues in the left half plane and $n$ eigenvalues in the right half plane.

For $\lambda=0$ system (15) takes the form:

$$
\begin{aligned}
& u_{1}^{\prime}=z_{1}, \\
& \ldots \ldots \\
& u_{n}^{\prime}=z_{n}, \\
& z_{1}^{\prime}=q z_{1}-g_{1}(u), \\
& \ldots \ldots \ldots \ldots \ldots \\
& z_{n}^{\prime}=q z_{n}-g_{n}(u) .
\end{aligned}
$$


To use the Conley connection index theory developed e.g. in [4] system (15) should be supplemented by an additional equation for $q$ :

$$
q^{\prime}=\rho \phi(u, z) q
$$

where $\rho$ is sufficiently small positive parameter. Let $W^{-}$and $W^{+}$denote open neighbourhoods in $\mathbb{R}^{2 n} \times(-Q-\varepsilon, Q+\varepsilon)$ of $(\mathbf{0}, \mathbf{0}) \times(-Q) \cup(\mathbf{0}, \mathbf{0}) \times Q$ and $(\mathbf{1}, \mathbf{0}) \times(-Q) \cup(\mathbf{1}, \mathbf{0}) \times Q$ respectively having disjoint closures. The real valued continuous function $\phi$ is arbitrary except for the fact that it is positive on $W^{-}$ and negative on $W^{+}$(see [4]).

Now, for $\lambda \in[0,1]$, let $I_{\lambda}^{-}:=(\mathbf{0}, \mathbf{0}) \times[-Q, Q], I_{\lambda}^{+}:=(\mathbf{1}, \mathbf{0}) \times[-Q, Q]$. According to Lemma p. 325 in [4] or due to the results in [3] for $\rho>0$ sufficiently small and all $\lambda \in[0,1]$ the set $N_{\lambda} \times[-Q, Q]$ is an isolating neighbourhood with respect to the flow generated by system (15) and equation (34).

REMARK 8. The last fact may be also proved straightforwardly. For all $\lambda \in[0,1]$ one may take $\phi$ in such a way that $\left.\phi\right|_{N_{\lambda} \times Q}$ is positive in some open $2 n$ dimensional ball $B_{1}$ of radius $r^{*}<\varepsilon / 2$ centered at $(\mathbf{0}, \mathbf{0}) \times Q$, negative in some open $2 n$-dimensional ball $B_{2}$ of radius $r^{*}$ centered at $(\mathbf{1}, \mathbf{0}) \times Q$ and equal to 0 elsewhere. Likewise, let $\left.\phi\right|_{N_{\lambda} \times(-Q)}$ be positive in some open $2 n$-dimensional ball $B_{3}$ of radius $r^{*}$ centered at $(\mathbf{0}, \mathbf{0}) \times(-Q)$, negative in some open $2 n$-dimensional ball $B_{4}$ of radius $r^{*}$ centered at $(\mathbf{1}, \mathbf{0}) \times(-Q)$ and equal to 0 elsewhere. Let us note that for $\rho>0$ sufficiently small invariant trajectories do not touch the set $\partial N_{\lambda} \times[-Q, Q]$, as it is true for $\rho=0$. Let us consider the set $N_{\lambda} \times Q$. Outside the balls $B_{1}$ and $B_{2}$ we have $\phi \equiv 0$, so the flow is the same as for $\rho=0$. Hence every trajectory through a point in $N_{\lambda} \times Q \backslash\left(B_{1} \cup B_{2}\right)$ lies in the plane $q=Q$ unless it reaches $B_{1}$ or $B_{2}$. Thus, it either leaves the set $N_{\lambda} \times Q$ or gets inside $B_{1}$ or $B_{2}$. However, the second possibility cannot take place, because a trajectory through every point inside $B_{1}$ or $B_{2}$ leaves $N_{\lambda} \times[-Q, Q]$ immediately in forward or backward direction. The same considerations hold for $N_{\lambda} \times(-Q)$. It follows that the set $N_{\lambda} \times[-Q, Q]$ is an isolating neighbourhood.

Let $I_{\lambda}$ denote the maximal invariant set in $N_{\lambda} \times[-Q, Q]$ with respect to the flow generated by (15) together with the equation $q^{\prime}=0$. Due to the results of the above sections the connection triples $\left(I_{\lambda}^{-}, I_{\lambda}^{+}, I_{\lambda}\right), \lambda \in[0,1)$, and $\left(I_{1}^{-}, I_{1}^{+}, I_{1}\right)$ are related by continuation. By Theorem in $\S 2$.D of [4] these triples have the same (homotopic) connection indices. According to the definition (see [4]) the connection index of the triple $\left(I_{\lambda}^{-}, I_{\lambda}^{+}, I_{\lambda}\right)$ is the Conley index of $N_{\lambda} \times[-Q, Q]$ with respect to the flow generated by (15) together with (34). This index is homotopic to the index of the maximal invariant set contained in $N_{0} \times[-Q, Q]$ with respect to the flow generated by system (33), (34).

Let us introduce the new variables $\zeta_{1}=u_{1}, \zeta_{i}=u_{i}-u_{1}, i=2, \ldots, n$. This transformation is a homeomorphism, so it does not change the homotopy 
class of the quotient spaces. In these variables the isolating neighbourhood $N_{0}$ is changed to the set $N_{\zeta}$ and the system (33)-(34) changes to

$$
\begin{aligned}
& \zeta_{1}^{\prime}=\theta_{1}, \\
& \ldots \ldots \\
& \zeta_{n}^{\prime}=\theta_{n}, \\
& \theta_{1}^{\prime}-q \theta_{1}+g_{1}^{*}=0, \\
& \theta_{2}^{\prime}-q \theta_{2}+g_{2}^{*} \zeta_{2}=0, \\
& \ldots \ldots \ldots \ldots \ldots \\
& \theta_{n}^{\prime}-q \theta_{n}+g_{n}^{*} \zeta_{n}=0,
\end{aligned}
$$

$$
q^{\prime}=\rho \phi^{*}(\zeta, \theta) q
$$

where $g_{1}^{*}=g_{1}(u(\zeta)), \phi^{*}=\phi(u(\zeta), z(\theta))$ and for $i=2, \ldots, n$, we have $g_{i}^{*} \zeta_{i}=$ $g_{1}\left(\zeta_{1}+\zeta_{i}, \ldots, \zeta_{1}+\zeta_{i-1}, \zeta_{1}, \ldots, \zeta_{1}+\zeta_{n}\right)-g_{1}\left(\zeta_{1}, \ldots, \zeta_{1}+\zeta_{i-1}, \zeta_{1}+\zeta_{i}, \ldots, \zeta_{1}+\zeta_{n}\right)=$ $\left\{g_{1}\left(\zeta_{1}+\zeta_{i}, \ldots, \zeta_{1}+\zeta_{i-1}, \zeta_{1}, \ldots, \zeta_{1}+\zeta_{n}\right)-g_{1}\left(\zeta_{1}, \ldots, \zeta_{1}+\zeta_{i-1}, \zeta_{1}, \ldots, \zeta_{1}+\zeta_{n}\right)\right\}-$ $\left\{g_{1}\left(\zeta_{1}, \ldots, \zeta_{1}+\zeta_{i-1}, \zeta_{1}+\zeta_{i}, \ldots, \zeta_{1}+\zeta_{n}\right)-g_{1}\left(\zeta_{1}, \ldots, \zeta_{1}+\zeta_{i-1}, \zeta_{1}, \ldots, \zeta_{1}+\zeta_{n}\right)\right\}=$ $g_{1,1}\left(\zeta_{* 1}\right) \zeta_{i}-g_{1, i}\left(\zeta_{* 2}\right) \zeta_{i}$ for some $\zeta_{* 1}, \zeta_{* 2} \in \mathbb{R}^{n}$. Due to Definition $2, g_{1,1}<0$ and $g_{1, i}>0$ for all $i=2, \ldots, n$. Thus, we conclude that for any bounded solution $\zeta(\xi)$ to system $(35)$

$$
\zeta_{2}(\xi)=\ldots=\zeta_{n}(\xi)=0
$$

for all $\xi \in \mathbb{R}^{1}$ (see Lemma 3.2 p. 173 in [18]). Hence, without changing the Conley index of the maximal invariant set in $N_{0} \times[-Q, Q]$ we can replace the last set of equations by

$$
\begin{aligned}
& \zeta_{1}^{\prime}=\theta_{1} \\
& \ldots \ldots \\
& \zeta_{n}^{\prime}=\theta_{n} \\
& \theta_{1}^{\prime}-q \theta_{1}+g_{1}\left(\zeta_{1}, \ldots, \zeta_{1}\right)=0 \\
& \theta_{2}^{\prime}-\zeta_{2}=0 \\
& \ldots \ldots \ldots \ldots \\
& \theta_{n}^{\prime}-\zeta_{n}=0
\end{aligned}
$$

and equation (36) by

$$
q^{\prime}=\rho \widetilde{\phi}\left(\zeta_{1}, \theta_{1}\right) q
$$

where $\widetilde{\phi}\left(\zeta_{1}, \theta_{1}\right)=\phi^{*}\left(\zeta_{1}, 0, \ldots, 0, \theta_{1}, 0, \ldots, 0\right)$. We note that each pair of $n-1$ equations in (38) for $\left(\zeta_{i}, \theta_{i}\right), i=2, \ldots, n$, is completely decoupled from the rest. 
Moreover, due to the robustness of the Conley index there is a homotopy $N_{\zeta}(\eta)$ such that

$$
N_{\zeta}(0)=N_{0}, \quad N_{\zeta}(1)=N_{\zeta}^{*} \times \underbrace{[-\delta, \delta]^{2} \times \ldots \times[-\delta, \delta]^{2}}_{(n-1) \text { times }},
$$

where $N_{\zeta}^{*}$ lies in the $\left(u_{1}, u_{1}^{\prime}\right)$-space, such that for $\eta \in[0,1]$ the set $N_{\zeta}(\eta)$ is an isolating neighbourhood for the flow generated by the system (38) for each $q \in[-Q, Q]$. Thus (cf. [2], [17]) the Conley index $h$ of $N_{\zeta}(1) \times[-Q, Q]$ with respect to the flow generated by system (38), (39) is homotopic to

$$
h_{1 \theta} \wedge \underbrace{h_{2} \wedge \ldots \wedge h_{n}}_{(n-1) \text { times }}
$$

where $h_{1 \theta}$ is the Conley index of $N_{\zeta}^{*} \times[-Q, Q]$ with respect to the flow generated by the pair of equations for $\zeta_{1}$ and $\theta_{1}$ together with (39), and $h_{i}, i=2, \ldots, n$, is the Conley index of the rectangle $[-\delta, \delta] \times[-\delta, \delta]$ with respect to the flow generated by the system

$$
\begin{aligned}
\zeta_{i}^{\prime} & =\theta_{i}, \\
\theta_{i}^{\prime}-\zeta_{i} & =0 .
\end{aligned}
$$

Obviously $h_{i} \cong \Sigma^{1}$. The index $h_{1}$ is computed in [4] and it is homotopic to $\overline{0}$. Thus

$$
h \cong \overline{0} \wedge \underbrace{\Sigma^{1} \wedge \ldots \wedge \Sigma^{1}}_{(n-1) \text { times }} \cong \overline{0} \wedge \Sigma^{n-1} \cong \overline{0} .
$$

For each $q=q_{0} \in[-Q, Q]$ the Conley index of the set $A=(\mathbf{0 , 0})$ and of the set $B=(\mathbf{1}, \mathbf{0})$ with respect to the flow generated by system (15) is equal to $\Sigma^{n}$. As $\left(\Sigma^{1} \wedge \Sigma^{n}\right) \vee \Sigma^{n}=\Sigma^{n+1} \vee \Sigma^{n}$ is not homotopic to $\overline{0}$, then according to Theorem of $\S 2 . \mathrm{F}$ in [4], it follows that $I_{1}^{-} \cup I_{1}^{+} \neq I_{1}$. Due to Lemma 11 we have thus proved the following existence theorem.

Theorem 1. Suppose that Assumptions 1-4 hold. Then there exists a heteroclinic pair $(q, u) \in(-Q, Q) \times C^{2}\left(\mathbb{R}^{1}\right)$ satisfying system $(3)$.

REMARK 9. As we mentioned the proof of Theorem 1 follows from Theorem of $\S 2$.F in [4]. However, for the reader's convenience, we will give a straightforward proof of it here. As a matter of fact one can prove that heteroclinic solutions exist for all $\lambda \in[0,1]$. Let us fix $\lambda \in[0,1]$. It is obvious that for $\rho>0$ sufficiently small the sets $\mathcal{B}(A, \varepsilon) \times[-Q, Q]$ and $\mathcal{B}(B, \varepsilon) \times[-Q, Q]$ are isolating neighbourhoods with respect to the flow generated by the system (15), (34) (see [3], [4] or use the arguments in Remark 8). The Conley index of $\mathcal{I}_{\rho}^{-}=I(\mathcal{B}(A, \varepsilon) \times[-Q, Q])$ with respect to the flow generated by system (15), (34) is homotopic to $\left(\Sigma^{1} \wedge \Sigma^{n}\right)$. Similarly, the Conley index of $\mathcal{I}_{\rho}^{+}=I(\mathcal{B}(B, \varepsilon) \times[-Q, Q])$ is homotopic to $\Sigma^{n}$. Finally, as it was noted above, the Conley index of $\mathcal{I}_{\rho}=I\left(N_{\lambda} \times[-Q, Q]\right)$ with 
respect to the flow generated by system (15) together with equation (34) is homotopic to $\overline{0}$. As $\left(\Sigma^{1} \wedge \Sigma^{n}\right) \vee \Sigma^{n}=\Sigma^{n+1} \vee \Sigma^{n}$ is not homotopic to $\overline{0}$, then $\mathcal{I}_{\rho} \neq \mathcal{I}_{\rho}^{-} \cup \mathcal{I}_{\rho}^{+}$(see e.g. [17, Theorem 22.31]). It follows that for all $\rho>0$ sufficiently small there exists at least one invariant trajectory, which is not contained completely neither in $\mathcal{B}(A, \varepsilon) \times[-Q, Q]$ nor in $\mathcal{B}(B, \varepsilon) \times[-Q, Q]$. Thus there exists at least one point on this trajectory contained in the set $N_{\lambda} \backslash(\mathcal{B}(A, \varepsilon) \times[-Q, Q] \cup \mathcal{B}(B, \varepsilon) \times[-Q, Q])$. According to Remark 6 the $u$ component of this solution is strictly monotone and $u(\rho, \xi) \rightarrow \mathbf{0}$ as $\xi \rightarrow-\infty$, $u(\rho, \xi) \rightarrow \mathbf{1}$ as $\xi \rightarrow \infty$ and $u^{\prime}(\rho, \xi) \rightarrow \mathbf{0}$ as $\xi \rightarrow \pm \infty$. So, all the invariant trajectories cross the plane $\left\{(u, z): u_{1}=1 / 2\right\}$ at exactly one point. Let us fix the position of the invariant trajectories for $\rho>0$ sufficiently small by the condition $u_{1}(\rho, 0)=1 / 2$. Let $\rho_{k}=1 / k$, where $k=1,2, \ldots$. For each $k$ let $\left(q_{k}(\xi), u_{k}(\xi), z_{k}(\xi)\right)$ denote a solution to system (15), (39) for $\rho=\rho_{k}$, with its values completely contained in the interior of $N_{\lambda} \times[-Q, Q]$. Let $\left\{n_{k}\right\}_{k=1}^{\infty}$ denote a subsequence of $\{k\}_{k=1}^{\infty}$ such that $\left\{q_{n_{k}}\right\}_{k=1}^{\infty}$ converges to some $q_{0}$. Out of this sequence we can in turn choose subsequences $\left\{n_{r k}\right\}_{k=1}^{\infty}$, where $r=1,2, \ldots$, having the property that the sequence $\left\{u_{n_{r k}}\right\}$ converges in $C^{2}([-r, r])$ to a function $u_{0 r}(\xi)$ uniformly in $\xi$ on the interval $[-r, r]$, and $\left\{n_{(r+1) k}\right\}_{k=1}^{\infty}$ is a subsequence of $\left\{n_{r k}\right\}_{k=1}^{\infty}$. (The convergence is guaranteed by Arzeli lemma and bootstrap argument.) Hence choosing the diagonal subsequence we obtain a sequence $\left\{m_{k}\right\}_{k=1}^{\infty}$ such that $\left\{u_{m_{k}}\right\}_{k=1}^{\infty}$ converges in $C^{2}$ to a function $u_{0}(\xi)$ uniformly in $\xi$ on every interval $[-r, r]$. Now, due to the strict monotonicity of $u_{k}(\xi)$ we conclude that $\left(q_{0}, u_{0}(\xi), z_{0}(\xi)\right)$ is a monotone heteroclinic triple for system (15) (and $\left(q_{0}, u_{0}(\xi)\right)$ is a monotone heteroclinic pair for system (10)). Using the fact that $\left(u_{0}(\xi), z_{0}(\xi)\right) \in N_{\lambda} \times q_{0}$ for all $\xi \in \mathbb{R}^{1}$ and Lemma 11 we conclude that $u_{0}: \mathbb{R}^{1} \rightarrow \mathbb{R}^{n}$ is strictly monotonic. It is worthwile to note that from the geometric point of view the limit trajectory may consist of the two parts: the image of the heteroclinic solution $\left(q_{0}, u_{0}, z_{0}\right)$ and of the singular segments lying on $I_{\lambda}^{-}$ and $I_{\lambda}^{+}$.

\section{Travelling waves in systems describing mutualist species}

As we noted in Introduction, equations (3) may describe travelling waves in ecological systems of mutualist type (see e.g. [14], [15], [9]). Then the functions $f_{i}$ become more specific, i.e. $f_{i}(u)=u_{i} \Phi_{i}\left(u_{i}\right) \widetilde{f}_{i}(u), i=1, \ldots, n$. We impose the following conditions on the functions $\widetilde{f}_{i}$ and $\Phi_{i}$, which are generalizations of conditions $\mathrm{H} 1-\mathrm{H} 5$ in [14].

Assumption 5. Assume that

(a) The functions $\widetilde{f}_{i}$ satisfy the conditions of local monotonicity, i.e.

$$
\tilde{f}_{i, j}(u)>0 \quad \text { for all } i, j \in\{1, \ldots, n\}, j \neq i \text {, and all } u \in[0,1]^{n}
$$


such that $\widetilde{f}_{i}(u)=0$.

(b) $\mathbf{1}=(1, \ldots, 1)$ is a solution to the system

$$
\tilde{f}_{i}(u)=0, \quad i=1, \ldots, n .
$$

This solution is stable, i.e. all the eigenvalues of the matrix

$$
\widetilde{f}_{i, j}(\mathbf{1})
$$

have negative real parts.

(c) All the other solutions $\left\{E_{1}, \ldots, E_{K}\right\}$ to system (4) not equal to $\mathbf{0}$ and $\mathbf{1}$ are contained in the interior of the set $[0,1]^{n}$ and are unstable i.e. the matrix $f_{i, j}\left(E_{k}\right), k=1, \ldots, K$, has at least one eigenvalue with positive real part.

(d) $\widetilde{f}_{i}(\mathbf{0})<0$.

(e) $\Phi\left(u_{i}\right)>0$ for all $u_{i} \in \mathbb{R}^{1}, u_{i} \neq 0$.

It is obvious that the negativity of the Perron-Frobenius eigenvalue of the matrix $\widetilde{f}_{i, j}(\mathbf{1})$ is equivalent to the negativity of the Perron-Frobenius eigenvalue of the matrix $f_{i, j}(\mathbf{1})$. One can also see that the positivity of the Perron-Frobenius eigenvalue of the matrix $f_{i, j}\left(E_{k}\right), k=1, \ldots, K$, is equivalent to the positivity of the Perron-Frobenius eigenvalue of the matrix $\widetilde{f}_{i, j}\left(E_{k}\right)$.

The functions $f$ satify the local monotonicity assumption only in the set $u>\mathbf{0}$. However, the methods of the previous sections can be used also in this case. The idea of the existence proof of heteroclinic solutions in this case is practically the same as in the previous section, yet relevant changes are necessary at some points. First, the functions $G_{\lambda}$ near the point $\mathbf{0}$ will be defined in a slightly different way. Let

$$
\omega_{*}(u)=w(|u|), \quad \omega^{*}(u)=w(|\mathbf{1}-u|),
$$

where the function $w$ is given by (11). $G_{\lambda}$ is defined formally as before

$$
G_{\lambda}(u)= \begin{cases}f(u) \omega_{\lambda}(u) & \text { for } \lambda \in[2 / 3,1], \\ 3(2 \lambda-1) f(u) \omega(u)+2(2-3 \lambda) h(u) & \text { for } \lambda \in[1 / 2,2 / 3], \\ 3(1-2 \lambda) g(u) \omega(u)+2(3 \lambda-1) h(u) & \text { for } \lambda \in[1 / 3,1 / 2], \\ g(u) \omega_{1-\lambda}(u) & \text { for } \lambda \in[0,1 / 3],\end{cases}
$$

where $\omega_{\lambda}(u)$ is defined by (12), but now

$$
h(u)=\omega_{*}(u)(-p I) u+\omega^{*}(u) H(u-\mathbf{1}) .
$$

$H$ is subject to the condition $H<D f(\mathbf{1}), D g(\mathbf{1})$ and $p>0$ is such that $-p I<$ $D f(\mathbf{0}), D g(\mathbf{0})$. Thus near $\mathbf{1}$ the $G_{\lambda}$ is the same as before and near $\mathbf{0}$ the matrix $H$ is replaced by a negative diagonal matrix $-p I$. 
LEMma 25. For all $\lambda \in[0,1]$ all the eigenvalues of the matrix $D G_{\lambda}(\mathbf{1})$ have negative real parts. For all $\lambda \in[0,1 / 3)$ the functions $G_{\lambda}(u)$ satisfy the conditions of local monotonicity. For $\lambda \in(2 / 3,1]$ the functions $G_{\lambda}(u)$ satisfy the conditions of local monotonicity for all $u \in[0,1]^{n}, u>\mathbf{0}$. Moreover, for $\delta>0$ sufficiently small, $G_{\lambda}(u)$ satisfies the condition of local monotonicity for all $\lambda \in[0,1]$ and all $u$ such that $|u-\mathbf{1}|<\delta$ or $\mathbf{0}<u<\delta \mathbf{1}$. The only zero of $G_{\lambda}(u)$ for $|u-\mathbf{1}|<\delta$ is $\mathbf{1}$.

Near the point $\mathbf{0}$ the following lemmas are also true.

Lemma 26. For all $\lambda \in[1 / 2,2 / 3]$ and for all $\delta>0$ sufficiently small the only solution to the equation

$$
\omega_{*}(u)[3(2 \lambda-1) f(u)-2(2-3 \lambda) p I u]=0
$$

in the ball $|u|<\delta$ is $\mathbf{0}$.

Proof. Let $2(2-3 \lambda)=l$. As $\omega_{*}(u)>0$ for $|u|<\delta$, then it suffices to consider the system

$$
u_{i}\left[(1-l) \Phi\left(u_{i}\right) \tilde{f}_{i}(u)-l p\right]=0, \quad i=1, \ldots, n .
$$

According to Assumption 5(d) $\widetilde{f}_{i}(u)<0$ for $|u|$ suffciently small, so for all $l \in[0,1]$ all the solutions $u$ from the ball $|u|<\delta$ to the $i$-th equation lie in the $\operatorname{disc}\left\{u:|u|<\delta, u_{i}=0\right\}$. The intersection of these sets for all $i$ is just the point $\mathbf{0 .}$

Lemma 27. For all $\lambda \in(1 / 3,1 / 2)$ the Perron-Frobenius eigenvalue of the matrix $[3(1-2 \lambda) D g(\mathbf{0})-2(3 \lambda-1) p I]$ is smaller than $\mu_{P F}(D g(\mathbf{0}))$. For all $\lambda \in[1 / 3,1 / 2]$ and for all $\delta>0$ sufficiently small the only solution to the equation

$$
\omega_{*}(u)[3(1-2 \lambda) g(u)-2(3 \lambda-1) p I u]=\mathbf{0}
$$

in the ball $|u|<\delta$ is $\mathbf{0}$.

Proof. The lemma may be proved as Lemma 4.

One notes that Lemma 5 holds also, when $f$ satisfies Assumption 5 instead of Assumption 2. The counterparts of Lemmas 6-8 hold also, but near $u=\mathbf{0}$ Lemma 6 must be reformulated because for $\lambda \geq 1 / 2$ the matrix $D G_{\lambda}(\mathbf{0})$ has its off-diagonal entries equal to zero and we cannot use Lemma 1.

LEMma 28. Let $f \in C^{1}\left(\mathbb{R}^{n}, \mathbb{R}^{n}\right)$ satisfy Assumption 5. Then for all $\lambda \in[0,1]$ the statements of Lemmas 6-8 hold with respect to functions $G_{\lambda i}$ defined by (42) near $u=\mathbf{1}$ with numbers $r$ and $\vartheta(r)$ independent of $\lambda$. The same is true near $u=\mathbf{0}$ for $\lambda \in[0,1 / 3)$. For $\lambda \in[1 / 3,1]$ there exist a number $\widetilde{\vartheta}>0$ and a continuous family of closed parallelepipeds $\widetilde{W}_{\lambda}=\left[0, w_{\lambda 1}\right] \times \ldots \times\left[0, \ldots, w_{\lambda n}\right]$ with positive sides such that $\widetilde{W}_{\lambda}=[0, w]^{n}, w>0$, for $\lambda \in[2 / 3,1]$, and such 
that for all $i$ we have $G_{\lambda i}(u)<0$ if $\operatorname{dist}\left(u, \widetilde{W}_{\lambda i}\right)<\widetilde{\vartheta}$, where $\widetilde{W}_{\lambda i}=\widetilde{W}_{\lambda} \cap\{u$ : $\left.u_{i}=w_{\lambda i}\right\}$. Moreover, for each $\lambda \in[1 / 3,1]$, the statement of Lemma 8 holds for $\widetilde{\delta}<1 / 2 \min _{j} w_{\lambda j}$.

Proof. The proof follows from Assumption 5, the definition of functions $G_{\lambda i}((42))$ and the proofs of Lemmas 6-8.

By means of the last lemma and Lemma 5 we can prove a priori estimates for the parameter $q$.

Lemma 29. If $\lambda \in[0,1]$ and $\left(q_{\lambda}, u_{\lambda}\right)$ is a heteroclinic pair satisfying system (10), with $f$ satisfying Assumption 5 instead of Assumption 2, such that $u_{\lambda}^{\prime}(\xi)>\mathbf{0}$ for all $\xi \in \mathbb{R}^{1}$, then the value of $\left|q_{\lambda}\right|<Q$, where $Q$ is independent of $\lambda$ and $u_{\lambda}$.

Lemma 30. Suppose, for $\lambda \in[0,1 / 3) \cup(2 / 3,1]$, that $\left(u_{0}, z_{0}\right) \in I(N) \cap \partial N$. Then $\left(u_{0}, z_{0}\right)$ is a singular point. The same holds also for $\lambda \in[1 / 3,2 / 3]$ if $\left|\mathbf{1}-u_{0}\right|<\delta$.

Proof. The proof can be carried out as the proof of Lemma 12 for $\lambda \epsilon$ $[0,1 / 3)$. So, let us take $\lambda \in(2 / 3,1]$. Below, for brevity, we will not discern the situation, when $|\xi|$ is finite or infinite at the considered points. Suppose that $u_{0 i}=0$ or $u_{0 i}=1$ for some point lying on a trajectory belonging to $I(N)$ and some $i \in\{1, \ldots, n\}$. Then we must have also $z_{0 i}=0$ at this point. Thus, the problem is reduced to examining these parts of $\partial N$ at which $z_{i}=0$ for $i \in\{1, \ldots, n\}$. So, let $z_{0 i}=0$ for some point lying on a trajectory belonging to $I(N)$. Then we must have also $z_{i}^{\prime}=0$ and $z_{i}^{\prime \prime} \geq 0$ at this point for the trajectory passing through $\left(u_{0}, z_{0}\right)$. From the $i$-th equation it follows that

$$
z_{i}^{\prime}=0=-G_{\lambda i}(u)=-\omega_{\lambda}(u) u_{i} \Phi_{i}\left(u_{i}\right) \widetilde{f}_{i}(u) .
$$

There are two possibilities. Either $u_{0 i}=0$ or $\widetilde{f}_{i}\left(u_{0}\right)=0$. Suppose that the first possibility takes place. Then the considered trajectory lies in the invariant plane $\left\{(u, z): u_{i}=0, z_{i}=0\right\}$. But as $z_{j} \geq 0$ in $N$, then for any $j=1, \ldots, n, j \neq i$, there must be at least one point on it, where $z_{j}=0$. At this point the two possibilities repeat (i.e. either $u_{j}=0$ or $\widetilde{f}_{i}\left(u_{1}, \ldots, u_{i-1}, 0, u_{i+1}, \ldots, u_{n}\right)=0$ ). As a result the considered trajectory lies in the invariant plane $Z_{\widetilde{K}}=\{(u, z)$ : $\left.u_{k}=0, z_{k}=0, k \in \widetilde{K}\right\}$, where $\widetilde{K}$ is a subset of $\{1, \ldots, n\}$. If $\widetilde{K}=\{1, \ldots, n\}$, then the considered trajectory reduces to a point $(\mathbf{0}, \mathbf{0})$. If $\widetilde{K}$ is empty then we can prove (as in the proof of Lemma 12) that $\left(u_{0}, z_{0}\right)$ is a singular point $\left(u_{0}, \mathbf{0}\right)$, with $\widetilde{f}\left(u_{0}\right)=\mathbf{0}$. Now, suppose that $\widetilde{K} \neq\{1, \ldots, n\}$, yet it is not empty. This situation is impossible. To show that, we note as above, that there exists a point with $z_{j}=z_{j}^{\prime}=\widetilde{f}_{j}=0, u_{j} \neq 0, j \notin \widetilde{K}$. By differentiating the $j$-th equation we obtain at this point the equality $u_{j} \Phi_{j}\left(u_{j}\right) \sum_{k \neq j, k \notin \widetilde{K}} \widetilde{f}_{j, k}(u) z_{k}=0$. As in 
the proof of Lemma 12 it follows from the local monotonicity conditions that all $z_{k}=0$ and all $f_{k}(u)=0, k \notin \widetilde{K}$. So this point would be a solution to the equation $f=\mathbf{0}$. But according to Assumption $5(\mathrm{c})$ there is no solution to the equation $f=\mathbf{0}$ at the faces of the set $[0,1]^{n}$ except for $\mathbf{0}$ and $\mathbf{1}$. The proof of the second claim can be carried out as the proof of Lemma 12.

Lemma 31. Suppose that Assumption 5 holds instead for Assumption 2. For all $\lambda \in[0,1]$ and all $q \in[-Q, Q]$ the point $(\mathbf{1}, \mathbf{0})$ is a saddle singular point. The same is true for the point $A=(\mathbf{0}, \mathbf{0})$ for all $\lambda \in[0,2 / 3)$ and all $q \in[-Q, Q]$.

ProOF. As the monotonicity conditions are satisfied near $u=\mathbf{1}$ the first claim follows from Theorem 3.3 in [5]. The same arguments hold for the point $A=(\mathbf{0}, \mathbf{0})$ if $\lambda \in[0,1 / 2)$. Now, note that the eigenvalues of the linearization matrix near $A=(\mathbf{0}, \mathbf{0})$ are solutions to the reduced eigenvalue problem:

$$
\left[a_{\lambda}(\mathbf{0}, 0) y^{2}-\left(q c_{\lambda}(\mathbf{0}, \mathbf{0})-M(\mathbf{0}, \mathbf{0})\right) y+D G_{\lambda}(\mathbf{0})\right] r=\mathbf{0},
$$

where

$$
\begin{aligned}
& a_{\lambda}(\mathbf{0}, 0)=\operatorname{diag}\left(a_{\lambda 1}(\mathbf{0}, 0), \ldots, a_{\lambda n}(\mathbf{0}, 0)\right), \\
& c_{\lambda}(\mathbf{0}, \mathbf{0})=\operatorname{diag}\left(c_{\lambda 1}(\mathbf{0}, \mathbf{0}), \ldots, c_{\lambda n}(\mathbf{0}, \mathbf{0})\right), \\
& M(\mathbf{0}, \mathbf{0})=\operatorname{diag}\left(M_{1}(\mathbf{0}, \mathbf{0}), \ldots, M_{n}(\mathbf{0}, \mathbf{0})\right),
\end{aligned}
$$

whereas $r \in \mathbb{R}^{n}$ is the reduced eigenvector (see [5 p. 1623]). If $\lambda \in[1 / 2,2 / 3$ ) then according to (42) $D G_{\lambda}(\mathbf{0})$ is also diagonal with negative entries, so the solutions to the above equation are real and nonzero. To be more precise, there are $n$ positive and $n$ negative values of $y$ satisfying the above eigenvalue problem.

LEMma 32. For all $\lambda \in[0,1], q \in[-Q, Q]$ with $Q$ as in Lemma 29 and $\varepsilon_{0}>0$ sufficiently small, no trajectory of a solution to system (10) (with $f$ satisfying Assumption 5 instead of 2$)$ belonging to $I\left(N^{*}\right)$ can have common points with the set $N^{*} \backslash N$.

Proof. It is obvious that near the point $B$ this lemma may be proved as Lemma 11. We will prove its validity near the point $A$. It is easy to note that this lemma holds for $\lambda \in[0,1 / 3]$. It also holds for $\lambda \in(1 / 2,1]$. The proof is almost the same as in [14], but for the reader's convenience we will insert it in the paper. Let us note that for these values of $\lambda$ for all $i$ we have $G_{\lambda i}(u)=u_{i} \widetilde{\Phi}_{\lambda i}\left(u_{i}\right) \widetilde{G}_{\lambda i}(u)$, where $\widetilde{G}_{\lambda i}(u)<0$ near $u=\mathbf{0}, \widetilde{\Phi}_{\lambda i}\left(u_{i}\right)>0$ for $u_{i} \neq 0$. From the proof of Lemma 30 it is clear that a trajectory belonging to $I\left(N^{*}\right)$ cannot have common points with the boundary $\partial N$ outside the balls $\mathcal{B}(A, \varepsilon)$. Suppose that there exists a trajectory $(u(\xi), z(\xi))$ from $I\left(N^{*}\right)$ such that $u_{i}\left(\xi_{*}\right)<0$ for some $\xi_{*}$ and some index $i$. We have two possibilities. First, suppose that there exists a finite $\xi_{0}$ such that $u_{i}(\xi)$ attains minimum at $\xi_{0}$ hence $u_{i}\left(\xi_{0}\right)<0, z_{i}\left(\xi_{0}\right)=0$ and 
$z_{i}^{\prime}\left(\xi_{0}\right) \geq 0$. This possibility is to be rejected as according to the $i$-th equation $a_{\lambda_{i}}\left(u\left(\xi_{0}\right), z_{i}\left(\xi_{0}\right)\right) z_{i}^{\prime}\left(\xi_{0}\right)=-u_{i}\left(\xi_{0}\right) \widetilde{\Phi}_{\lambda i}\left(u\left(\xi_{0}\right)\right) \widetilde{G}_{\lambda i}\left(u_{i}\left(\xi_{0}\right)\right)<0$. If such $\xi_{0}$ does not exist then $u_{i}(\xi) \geq \lim _{\xi \rightarrow \infty} u_{i}(\xi)$ for $\xi \in\left[\xi_{*}, \infty\right)$ or $u_{i}(\xi) \geq \lim _{\xi \rightarrow-\infty} u_{i}(\xi)$ for $\xi \in\left(-\infty, \xi_{*}\right]$. In this case $z_{i}(\xi) \leq 0$ for $\xi \in\left(\xi_{*}, \infty\right)$ or $z_{i}(\xi) \geq 0$ for $\xi \in\left(-\infty, \xi_{*}\right)$ respectively. (Otherwise $z_{i}(\xi)$ would change its sign and $u_{i}(\xi)$ would attain a minimum for finite $\xi$.) Consequently, $z_{i}(\xi) \rightarrow 0$ as $\xi \rightarrow \infty$ or $\xi \rightarrow-\infty$. Hence we would have $a_{\lambda_{i}}\left(u(\xi), z_{i}(\xi)\right) z_{i}^{\prime}(\xi)=-u_{i}(\xi) \widetilde{\Phi}_{\lambda i}(u(\xi)) \widetilde{G}_{\lambda i}(u(\xi))+o(1)<0$ as long as we are in the ball $\mathcal{B}(A, \varepsilon)$. But this contradicts the fact that $z_{i}(\xi) \rightarrow 0$. Thus we must have $u_{j} \geq 0$ for all $j$. Now, suppose that for an invariant trajectory we have $z_{i}\left(\xi_{0}\right)<0, u_{i}\left(\xi_{0}\right) \geq 0$ for some finite $\xi_{0}$. We will prove that $z_{i}(\xi)<0$ as long as $\xi<\xi_{0}$ is such that $\widetilde{G}_{\lambda i}(u(\xi))<0$. For, suppose that $z_{i}(\widetilde{\xi})=0$ and $z_{i}(\xi)<$ 0 for all $\xi \in\left(\widetilde{\xi}, \xi_{0}\right)$. Then $a_{\lambda_{i}}\left(u(\widetilde{\xi}), z_{i}(\widetilde{\xi})\right) z_{i}^{\prime}(\widetilde{\xi})=-u_{i}(\widetilde{\xi}) \widetilde{\Phi}_{\lambda i}\left(u_{i}\right) \widetilde{G}_{\lambda i}(u(\widetilde{\xi}))>0$, if $\widetilde{G}_{\lambda i}(u(\widetilde{\xi}))<0$. (Note that $u_{i}\left(\xi_{0}\right) \geq 0$ hence $u_{i}(\widetilde{\xi})>0$.) This leads to a contradiction. (The same arguments hold, if $z_{i}(\xi) \rightarrow 0$ as $\xi \rightarrow-\infty$.) So, the backward trajectory starting from the point $\left(u\left(\xi_{0}\right), z\left(\xi_{0}\right)\right)$ would leave the region, where $\widetilde{G}_{\lambda i}(u)<0$ and consequently the ball $\mathcal{B}(A, \varepsilon)$. (We assume that $\varepsilon_{0}<\delta$ is sufficiently small.) But a point $(u, z) \notin \mathcal{B}(A, \varepsilon)$ with $z_{i}<0$ does not belong to $N^{*}$.

Thus for $\lambda \in(1 / 2,1]$ the lemma is proved, as we have come to the conclusion that invariant trajectories satisfy in $\mathcal{B}\left(A, \varepsilon_{0}\right)$ the conditions:

$$
\begin{aligned}
& u_{j} \equiv 0, z_{j} \equiv 0, \quad j \in \widetilde{K}, \\
& u_{j}>0, z_{j}>0, \quad j \in\{1, \ldots, n\} \backslash \widetilde{K},
\end{aligned}
$$

where $\widetilde{K} \subset\{1, \ldots, n\} ; \widetilde{K}$ may be empty.

Now we will show that Lemma 32 also holds for $\lambda \in(1 / 3,1 / 2]$. For these values of $\lambda$ the system (10) takes the form

$$
u_{i}^{\prime \prime}-q u_{i}^{\prime}+G_{\lambda i}(u)=0, \quad i=1, \ldots, n .
$$

Let $3(1-2 \lambda)=l$. Take any $l \in[0,1]$. Proceeding as in the proof of Lemma 11 we arrive at the equation corresponding to (24):

$$
v_{i}^{\prime \prime}-q v_{i}^{\prime}-(1-l) p v_{i}+l \sum_{j} g_{i, j}(u) v_{j}=0 .
$$

Let $\varepsilon_{0}<\delta / 2$ be so small that for $|u|<\varepsilon_{0}$ the following conditions are satisfied:

(1) $D g(u)$ has all of its off-diagonal terms positive,

(2) $D g(u) P(\mathbf{0})<\mathbf{0}$.

According to Definition 2 and Remark 4, it is obvious that the constant $\varepsilon_{0}$ with the above mentioned properties exist and is independent of $l$ and hence of $\lambda$. 
If $\xi^{*}$ has the same meaning as in the proof of Lemma 11 and $\left|u\left(\xi^{*}\right)\right|<\varepsilon_{0}$ then one can show that (for all $i$ )

$$
-(1-l) p v_{i}\left(\xi^{*}\right)+l \sum_{j} g_{i, j}\left(u\left(\xi^{*}\right)\right) v_{j}\left(\xi^{*}\right)>0 .
$$

If $l=0$ then it is obviously true as $p>0$. For $l \in(0,1]$ the proof of this inequality can be done as in the proof of Lemma 11. In both cases we arrive at a contradiction (as in the proof of Lemma 11).

REMARK 10. As in the case of Lemma 11 it is seen that this proof is valid also in the case, when $q$ is not a constant parameter, but varies suffficiently slowly, e.g. if $q^{\prime}=\rho \phi(u, z) q$, where $\phi$ is globally bounded and $\rho>0$ is a parameter, which can be taken arbitrarily small. Near the point $B$ and near the point $A$ for $\lambda \in[0,1 / 3]$ arguments corresponding to Remark 6 hold. Near the point $A$ and for $\lambda \in(1 / 2,1]$ the proof of Lemma 32 may be repeated. For $\lambda \in(1 / 3,1 / 2]$ we may use the arguments from Remark 6 to state that if a segment of an invariant trajectory is contained in $\mathcal{B}(A, \varepsilon) \backslash N$ then there must exist an index $i$ such that $z_{i}$ is negative for some $\xi$. Then (43) changes to

$$
v_{i}^{\prime \prime}-q v_{i}^{\prime}-(1-l)\left(p+\rho q c_{\lambda i} \phi\right) v_{i}+l \sum_{j}\left(g_{i, j}(u)-\rho q c_{\lambda i} \phi \delta_{i j}\right) v_{j}=0 .
$$

It is seen that the proof can be completed as the proof of Lemma 13.

Now, we will prove a lemma corresponding to Lemma 13.

Lemma 33. For $\lambda \in[1 / 3,2 / 3]$, suppose that $(U, Z) \in I(N) \cap \partial N$ and $|U|<\delta$. Then, if $q \geq 0$, the trajectory passing through $(U, Z)$ must coincide with the singular point $(\mathbf{0}, \mathbf{0})$. If $q<0$ then it tends to a point in $\mathcal{P}$ defined in Definition 5.

Proof. Let $\lambda \in(1 / 2,2 / 3]$. As above it suffices to consider the points $(u, z)$ on the trajectories from $I(N)$ with $z_{i}=0$ for some $i$. If $u$-coordinates of the points on a trajectory from $I(N)$ stay inside the set $|u|<\delta$ then, as in the proof of Lemma 30, one can show that $(U, Z)=(\mathbf{0}, \mathbf{0})$. (Remind that the only singular point $(u, \mathbf{0})$ with $|u|<\delta$ is $(\mathbf{0}, \mathbf{0})$.) Suppose that the considered trajectory leaves the set $\{(u, z):|u|<\delta\}$. From the proof of Lemma 32 it follows that we must have $U_{i}=Z_{i}=0$. So, this trajectory lies in the invariant plane $\left\{(u, z): u_{i}=0, z_{i}=0\right\}$. It may tend to the point $(u, \mathbf{0})$ with $|u|=\delta$ or leave the set $\{(u, z):|u| \geq \delta\}$. Suppose first that it leaves this set and crosses its boundary at a point $(u, z)$, but stays in $N$. Then at this point we must have $z_{j}>0$ for at least one $j \in\{1, \ldots, n\}$. As we noted, the trajectory lies in the invariant space $\left\{(u, z): u_{i}=0, z_{i}=0\right\}$. As at the exit point we have $z_{k}^{\prime}=q z_{k}$ for all $k$, then for $q \geq 0$ it leaves the set $N^{*}$ and cannot belong to $I\left(N^{*}\right)$. For $q<0$ the trajectory either leaves $N^{*}$ or tends as $\xi \rightarrow \infty$ to one the points belonging to $\mathcal{P}$ (see the proof of Lemma 22). Now, suppose that the trajectory tends to 
the point $(u, \mathbf{0})$ with $|u|=\delta$ as $\xi \rightarrow \infty$. We will show that for $q \geq 0$ this is impossible. From the proof of Lemma 32 it follows that $z_{j}(\xi)>0$ for all $j \notin \widetilde{K}$ and all $\xi \in \mathbb{R}^{1}$. So, for all $j \notin \widetilde{K}$ there must exists a sequence $\left\{\xi_{J}^{j}\right\}_{J=1}^{\infty}$ such that $\xi_{J}^{j} \rightarrow \infty$ as $J \rightarrow \infty$ and $z_{j}^{\prime}\left(\xi_{J}^{j}\right)<0$. (Otherwise, $z_{j}(\xi)$ could not tend to 0 , because $z_{j}(\xi)>0$ and its derivative would be nonnegative for $\xi$ sufficiently large.) As $\left(\omega\left(u\left(\xi_{J}^{j}\right)\right)\right)^{-1} z_{j}^{\prime}\left(\xi_{J}^{j}\right)=-\left(\omega\left(u\left(\xi_{J}^{j}\right)\right)\right)^{-1} G_{\lambda j}\left(u\left(\xi_{J}^{j}\right)\right)+\left(\omega\left(u\left(\xi_{J}^{j}\right)\right)\right)^{-1} q z_{j}\left(\xi_{J}^{j}\right)$ and $u_{j}\left(\xi_{J}^{j}\right) \rightarrow U$ it follows that $G_{\lambda j}(U) \geq 0$. But, if $\delta$ is taken sufficiently small, then for $\lambda \in(1 / 2,2 / 3]$ we have $G_{\lambda j}(U)<0$ for all $U$ satisfying $|U| \leq \delta$ and $U_{j}>0$. This contradiction proves our claim.

For $\lambda=1 / 2$ the equations of system (15) become completely uncoupled, so the proof is obvious. For $\lambda \in[1 / 3,1 / 2)$, there are no invariant planes of the form $\left\{(u, z): u_{k}=0, z_{k}=0\right\}$. The functions $G_{\lambda i}(u)$ satisfy the monotonicity conditions, thus the proof may be carried out as the proof of the corresponding claims in Lemmas 21-23.

The remaining stages of the existence proof can be done as in the case of Assumption 2. In particular the excision of the sets of singular points occuring at the boundary of $N$ can be done in the same way as in the case of Assumption 2. The lemmas corresponding to Lemmas 21-23 read.

Lemma 34. Let Assumptions 5 hold instead of Assumption 2. For $\lambda \in$ $[1 / 3,2 / 3]$ and $q=0$ both $A_{+}(\lambda, q)$ and $A_{-}(\lambda, q)$ are empty and $I(N) \cap \partial N^{*}=\mathcal{P}$. Moreover, $\mathcal{P}$ is the maximal invariant set in the closure of a sufficiently small neighbourhood of $\mathcal{P}$ contained in $N$.

Lemma 35. Let Assumptions 5 hold instead of Assumption 2. For $\lambda \in$ $[1 / 3,2 / 3]$ and $q>0$ the set $A_{+}(\lambda, q)$ is empty. Moreover, $\mathcal{P}$ is the maximal invariant set in the closure of a sufficiently small neighbourhood of $\mathcal{P}$ contained in $N$.

Lemma 36. Let Assumptions 5 hold instead of Assumption 2. For $\lambda \in$ $[1 / 3,2 / 3]$ and $q<0$ the set $A_{-}(\lambda, q)$ is empty. Moreover, $\mathcal{P}$ is the maximal invariant set in the closure of a sufficiently small neighbourhood of $\mathcal{P}$ contained in $N$.

For $u \notin b(\mathbf{0}, \delta)$ the proofs of these lemmas can be carried out in the same way as the proofs of Lemmas 21-23. For $u \in b(\mathbf{0}, \delta)$ the trajectories can be analyzed as in the proof of Lemma 33.

If $\Phi_{i}(0)=0$ for at least one $i$, then for $\lambda \in[2 / 3,1]$ point $A=(\mathbf{0}, \mathbf{0})$ is not a hyperbolic singular point. However, the following statement is true.

Lemma 37. For all $\lambda \in[2 / 3,1], q \in[-Q, Q]$ the point $A=(\mathbf{0}, \mathbf{0})$ is an isolated invariant set. Its Conley index is equal to $\Sigma^{n}$. 
Proof. The first part of the lemma is implicitly contained in the proof of Lemma 32, but for the reader's convenience we will repeat the arguments here. Let us take $\varepsilon_{A}>0$ so small that $\widetilde{f}_{i}(u)<0$ for $|u|<\varepsilon_{A}$. Suppose that there exists an invariant trajectory contained completely in the ball $\{(u, z)$ : $\left.\sup (|u|,|z|)<\varepsilon_{A}\right\}$ Then for every $i$ the function $u_{i}(\xi)$ must attain its maximal and minimal value (for $|\xi|$ finite or infinite). At these points $z_{i}^{\prime}=-u_{i} \Phi \widetilde{f}_{i}$, thus $u_{i} \leq 0$ at the point of its maximum and $u_{i} \geq 0$ at the point of its minimum. In consequence this trajectory must coincide with the point $(\mathbf{0}, \mathbf{0})$. Thus the set $\left\{(u, z): \sup (|u|,|z|)<\varepsilon_{A}\right\}$ is an isolating neighbourhood. Moreover, this set is an isolating neighbourhood for the point $A$ also for all $\lambda \in[2 / 3-\Delta, 1]$ for some $\Delta>0$. Hence by decreasing $\varepsilon_{A}$ if necessary and by using Lemma 31 we can achieve that the ball $\mathcal{B}\left(A, \varepsilon_{A}\right)$ is an isolating neighbourhood of $A$ for all $\lambda \in[0,1], q \in[-Q, Q]$. The Conley indices of maximal invariant sets in the same isolating neighbourhood are homotopic (see e.g. [4], [13] and references therein). Hence by Lemmas 31 and 24 follows the second claim of the lemma.

We may thus state the following theorem:

Theorem 2. Suppose that Assumptions 1, 3-5 hold. Then there exists a heteroclinic pair $(q, u) \in(-Q, Q) \times C^{2}\left(\mathbb{R}^{1}\right)$ satsifying system (3).

\section{Ionization waves in laser plasma}

In this section we will consider a system of equations describing multicomponent plasma sustained by a laser beam of a given intensity $I$. By this we mean plasma created in gas consisting of $(n-1) \geq 1$ different components. Under a constant pressure $p$ the temperature $T_{1}$ of the light (electron) component and the temperatures $T_{i}, i \in\{2, \ldots, n\}$, of heavy particles (atoms and ions) of $i$-th kind are described by the following equations (see [7], [8], [11], [12], [16]):

$$
\begin{aligned}
\left(\frac{\partial}{\partial t}+\vec{v} \cdot \nabla\right)\left\{\frac{3}{2} k_{B} N_{1} T_{1}+\widetilde{E}\left(T_{1}\right)\right\} & =\nabla\left(k_{1} \nabla T_{1}\right)+f_{1}(T), \\
\left(\frac{\partial}{\partial t}+\vec{v} \cdot \nabla\right)\left\{\frac{3}{2} k_{B} N_{i} T_{i}\right\} & =\nabla\left(k_{i} \nabla T_{i}\right)+f_{i}(T),
\end{aligned}
$$

$i=2, \ldots, n$. Here $T=\left(T_{1}, \ldots, T_{n}\right), k_{j}=k_{j}(T), j \in\{1, \ldots, n\}$, is the heat conductivity coefficient, $N_{1}\left(T_{1}\right)$ is the number density of electrons, $N_{i}\left(T_{i}\right)$ is the number density of the heavy component of $i$-th kind and $\vec{v}(T)$ denotes the common convectional velocity. $k_{B}$ is the Boltzmann constant. $\widetilde{E}\left(T_{1}\right)$ is the average ionization energy for the given temperature $T_{1}$. (The energy necessary to the first ionization of an atom depends on the kind of the atom. If we have to do with a one component plasma, then $\widetilde{E}$ would be equal simply to $N_{1}\left(T_{1}\right) E$, where $E$ is the first ionization energy for the given kind of atoms.) The functions 
$f_{i}$ have the following form:

$$
\begin{aligned}
f_{1} & =F_{1}\left(T_{1}\right)+\sum_{j \in\{2, \ldots, n\}} c_{1 j}(T)\left(T_{j}-T_{1}\right), \\
f_{i} & =\sum_{j \in\{1, \ldots, n\}, j \neq i} c_{i j}(T)\left(T_{j}-T_{i}\right)+K_{i}(T),
\end{aligned}
$$

for $i=2, \ldots, n$. The term $F_{1}=\kappa\left(T_{1}\right) I-\mathcal{E}_{\text {rad }}\left(T_{1}\right)$ is responsible for the absorption of energy from the laser beam $(\kappa I)$ and its losses by through radiation $\left(\mathcal{E}_{\text {rad }}\right)$. The terms $K_{i}(T)$ describe the losses of energy in the process of heat conduction and convection. The terms $c_{i j}(T)\left(T_{j}-T_{i}\right)$ describe the transfer of energy form the $i$-th to the $j$-th component of the plasma.

Let us look for solutions in the form of travelling waves:

$$
T_{i}(x, t)=u_{i}(x \cdot \vec{n}+\chi t), \quad i=1, \ldots, n,
$$

where $\vec{n} \in \mathbb{R}^{3}$ is a chosen unit vector (the direction of propagation) and $\chi \in \mathbb{R}^{1}$ is the speed of the wave. If we denote $\xi:=x \cdot \vec{n}+\chi t$, then we arrive at a system of ordinary differential equations:

$$
\left(k_{i} u_{i}^{\prime}\right)^{\prime}-q C_{i}(u) u_{i}^{\prime}+f_{i}(u)=0,
$$

$i=1, \ldots, n$, where $u:=\left(u_{1}, \ldots, u_{n}\right)$ and

$$
q:=(\chi+\vec{v} \cdot \vec{n}) \rho(u), \quad C_{i}(u)=(\rho(u))^{-1} \frac{\partial}{\partial u_{i}}\left\{\frac{3}{2} k_{B} N_{i}\left(u_{i}\right) u_{i}+\delta_{i 1} \widetilde{E}\left(u_{i}\right)\right\},
$$

with $\delta_{i 1}$ being the Kronecker's delta. From the continuity equation for the plasma as a whole it follows that $q=$ const. To prove it, let us note that $\rho=\rho(u)$, and $\vec{v}=\vec{v}(u)$ can be treated as functions of $\xi=x \cdot \vec{n}+\chi$ t. Thus while differentiating these quantities we can put $\partial / \partial t=\chi \partial / \partial \xi$ and $\partial / \partial x_{k}=n_{k} \partial / \partial \xi$. Hence from the continuity equation we get $[\rho(\chi+\vec{v} \cdot \vec{n})]_{, \xi}=0$, which proves our claim. The quantity $q$ can be interpreted as the mass speed of the wave in the system of reference moving with the gas.

Assumption 6. The function $F_{1}\left(u_{1}\right)$ has exactly three zeros: 0,1 and $U_{0} \in$ $(0,1)$ such that $F_{1}^{\prime}(0)<0, F_{1}^{\prime}\left(U_{0}\right)>0$ and $F_{1}^{\prime}(1)<0$.

Assumption 7. $\sup _{i \in\{2, \ldots, n\}} \sup _{u \in[-1,2]}\left(\left|K_{i}(u)\right|+\left|D K_{i}(u)\right|\right)<\tau$ with $\tau$ sufficiently small. $K_{i}(\mathbf{0})=0$ for all $i \in\{2, \ldots, n\}$.

This assumption is reasonable, as both the absorption of energy (in the process of so called Inverse Bremsstrahlung) and the energetic losses are almost entirely carried out by the electron component. 
ASSUMPtion 8. $c_{i j}(u)>0, c_{i j}(u)=c_{j i}(u)$ for all $i, j \in\{1, \ldots, n\}, u \in$ $[0,1]^{n}, \sum_{j \neq i} c_{i j, k}(u)\left(u_{j}-u_{i}\right)+c_{i k}(u)>0$, for all $i, k \in\{1, \ldots, n\}, k \neq i$, and all $u \in[0,1]^{n}$.

The last part of this assumption may be justified by the fact that the derivatives $c_{i j, k}(u)$ are relatively large only for small values of $u$. Thus they are damped by the factors $\left(u_{i}-u_{j}\right)$.

Assumption 9. $C_{i}(u)>C_{0 i}>0$ for all $u \in[0,1]^{n}$.

As $C_{i}$ depend only on $u$, then in view of Assumption 9 system (49) satisfies Asumption 3 (the condition $a_{i}(u)>1$ for all $u \in[0,1]^{n}$ can be achieved by dividing the $i$-th equation by $\left.\min _{u \in[0,1]^{n}} a_{i}(u)\right)$. It also satifies point (c) of Asumption 4. Now, we will show that Assumptions 6-8 imply Assumption 2. We have for $i \neq 1, k \neq i$

$$
f_{i, k}(u)=\sum_{j \neq i} c_{i j, k}\left(u_{j}-u_{i}\right)+c_{i k}(u)+K_{i, k}(u),
$$

whereas for $i=1, k \neq 1$

$$
f_{1, k}(u)=\sum_{j \neq i} c_{1 j, k}\left(u_{j}-u_{i}\right)+c_{i k}(u) .
$$

From Assumption 8 it follows that for $\tau>0$ sufficiently small $f_{i, k}(u)>0$. Thus the monotonicity condition (see Assumption 2(a)) is satisfied. Also the other points of Assumption 2 are satisfied. To prove it we must examine the roots of the system (4) and the structure of eigenvalues of $D f$ at these roots. First, using the fact that the terms $K_{i}(u)$ were assumed sufficiently small, we will analyze the solutions to the simplified system of the form:

$$
\begin{aligned}
F_{1}\left(u_{1}\right)+\sum_{j \neq 1} c_{1 j}(u)\left(u_{j}-u_{1}\right) & =0 \\
\sum_{j \neq i} c_{i j}(u)\left(u_{j}-u_{i}\right) & =0
\end{aligned}
$$

where $i=2, \ldots, n$.

LEMMA 38. The only solutions to system $(50)$ are $(0, \ldots, 0),(1, \ldots, 1)$ and $\left(U_{0}, \ldots, U_{0}\right)$.

Proof. Adding the equations and using the symmetry $c_{i j}=c_{j i}$, we obtain:

$$
F_{1}\left(u_{1}\right)=0 .
$$

Hence the first component of the solution to system (50) is equal to one of the solutions to (51). The set of $n-1$ equations for $i=2, \ldots, n$ can be written in the form:

$$
\mathcal{M}_{n-1}\left(u_{2}, \ldots, u_{n}\right)^{T}=-u_{1}\left(c_{21}(u), \ldots, c_{n 1}(u)\right)^{T}
$$


where

$$
\mathcal{M}_{n-1}=\left(\begin{array}{cccc}
-\sum_{j \neq 2} c_{2 j}(u) & c_{23}(u) & \ldots & c_{2 n}(u) \\
c_{32}(u) & -\sum_{j \neq 3} c_{3 j}(u) & \ldots & c_{3 n}(u) \\
\ldots & \ldots & \ldots & \ldots \\
c_{n 2}(u) & c_{n 3}(u) & \ldots & -\sum_{j \neq n} c_{n j}(u)
\end{array}\right) .
$$

Consider an auxilliary matrix arising from $\mathcal{M}_{n-1}$ by rejecting from the diagonal sums the terms $c_{i 1}$, i.e.

$$
\left(\begin{array}{cccc}
-\sum_{j \neq 1,2} c_{2 j}(u) & c_{23}(u) & \ldots & c_{2 n}(u) \\
c_{32}(u) & -\sum_{j \neq 1,3} c_{3 j}(u) & \ldots & c_{3 n}(u) \\
\ldots & \ldots & \ldots & \ldots \\
c_{n 2}(u) & c_{n 3}(u) & \ldots & -\sum_{j \neq 1, n} c_{n j}(u)
\end{array}\right) .
$$

The Perron-Frobenius eigenvalue of this matrix is equal to 0 , whereas the eigenvector corresponding to this eigenvalue is equal to $(1, \ldots, 1)$. Using Lemma 3 we infer that all the eigenvalues of $\mathcal{M}_{n-1}$ will be negative, hence $\operatorname{det} \mathcal{M}_{n-1} \neq 0$. Thus system (52), for a given $u_{1}$ has exactly one solution. It is equal to $\left(u_{1}, \ldots, u_{1}\right)$, where $u_{1}$ satisfies the equation $F_{1}(y)=0$.

Now, let us find the structure of eigenvalues of $D f(\widetilde{u})$ for $\widetilde{u}$ equal to $(0, \ldots, 0)$, $(1, \ldots, 1)$ and $\left(U_{0}, \ldots, U_{0}\right)$. We have

$$
D f(\widetilde{u})=\left(\begin{array}{cccc}
F_{1}^{\prime}(\widetilde{u})-\sum_{j \neq 1} c_{1 j}(\widetilde{u}) & c_{12}(\widetilde{u}) & \ldots & c_{1 n}(\widetilde{u}) \\
c_{21}(\widetilde{u}) & -\sum_{j \neq 2} c_{2 j}(\widetilde{u}) & \ldots & c_{2 n}(\widetilde{u}) \\
\ldots & \ldots & \ldots & \ldots \ldots \\
c_{n 1}(\widetilde{u}) & c_{n 2}(\widetilde{u}) & \ldots & -\sum_{j \neq 1, n} c_{n j}(\widetilde{u})
\end{array}\right) .
$$

(Note that the terms proportional to $c_{i, k}(\widetilde{u})\left(\widetilde{u}_{i}-\widetilde{u}_{j}\right)$ vanish.) Let us consider the matrix:

$$
\mathcal{M}_{n}(\widetilde{u})=\left(\begin{array}{cccc}
-\sum_{j \neq 1} c_{1 j}(\widetilde{u}) & c_{12}(\widetilde{u}) & \ldots & c_{1 n}(\widetilde{u}) \\
c_{21}(\widetilde{u}) & -\sum_{j \neq 2} c_{2 j}(\widetilde{u}) & \ldots & c_{2 n}(\widetilde{u}) \\
\ldots & \ldots & \ldots & \ldots \ldots \\
c_{n 1}(\widetilde{u}) & c_{n 2}(\widetilde{u}) & \ldots & -\sum_{j \neq 1, n} c_{n j}(\widetilde{u})
\end{array}\right) .
$$

As before one notes that the Perron-Frobenius eigenvalue of this matrix is equal to 0 , whereas the eigenvector corresponding to this eigenvalue is equal to $(1, \ldots, 1)$. Thus by means of Lemma 3 we have proved the following lemma.

Lemma 39. All the eigenvalues of $D f(\widetilde{u})$ have their real parts negative, if $F_{1}^{\prime}\left(\widetilde{u}_{1}\right)<0$. If $F_{1}^{\prime}\left(\widetilde{u}_{1}\right)>0$, its Perron-Frobenius eigenvalue is positive.

Due to the implicit function theorem Lemma 38 can be replaced by the following one. 
LEMma 40. Assume that the function $F_{1}\left(u_{1}\right)$ has exactly three zeros: 0,1 and $u_{0} \in(0,1)$. Then the only solutions to system (4) (with $f$ given by $\left.(47)\right)$ are $(0, \ldots, 0),\left(\bar{u}_{1}, \ldots, \bar{u}_{n}\right)=(1, \ldots, 1)+O(\tau)$ and $\left(\widehat{u}_{1}, \ldots, \widehat{u}_{n}\right)=\left(U_{0}, \ldots, U_{0}\right)+$ $O(\tau)$.

By means of this lemma and the fact that the eigenvalues of a matrix depend continuously on parameters we may prove the lemma corresponding to Lemma 39.

LEMma 41. For $\tau$ sufficiently small all the eigenvalues of $D f(u)$, for $u=$ $\left(u_{1}, \ldots, u_{n}\right)$ equal to one of the solutions to system (4), have their real parts negative, if $F_{1}^{\prime}\left(u_{1}\right)<0$, whereas the Perron-Frobenius eigenvalue of $D f(u)$ is positive, if $F_{1}^{\prime}\left(u_{1}\right)>0$.

By the linear change of variables $u_{i} \rightarrow\left(\bar{u}_{i}\right)^{-1} u_{i}$ the largest root of system (47) becomes equal to $(1, \ldots, 1)$. Thus using Theorem 1 we can state the following result.

THEOREM 3. Suppose that all the functions in system (49) are suffciently smooth and that Assumption 6-9 are fulfilled. Then there exists $q^{*} \in \mathbb{R}^{1}$ such that for $q=q^{*}$ system (49) has a strictly monotone heteroclinic solution.

\section{Appendix. Proof of Lemma 20}

First we will prove the following auxilliary lemma.

LEMma 42. There exists an open neighbourhood $U$ of the set $\widehat{Z}$ in $\mathbb{R}^{2 n}$ such that $\partial(N \backslash U) \cap(\bar{U} \cap N)$ does not contain the points belonging to $I(N \backslash U)$.

Proof. Let $O^{*}$ be equal to a closed neighbourhood of $\widehat{Z}$ in $\mathbb{R}^{2 n}$ such that $\delta \leq \operatorname{dist}\left(\widehat{Z}, \partial O^{*}\right) \leq 2 \delta$. For $\delta$ sufficiently small we have $I\left(O^{*}\right)=\widehat{Z}$. Let $O_{\delta}=O^{*} \cap N$. Then $O_{\delta}$ is a closed relative neighbourhood of the set $\widehat{Z}$ in $N$ such that $\delta \leq \operatorname{dist}\left(\widehat{Z}, \partial O_{\delta} \backslash \partial N\right) \leq 2 \delta$ and $I\left(O_{\delta}\right)=\widehat{Z}$. Now, suppose that there does not exist $U$ satisfying the claim of the lemma. Then for every open neighbourhood $U_{\varepsilon} \subset \mathbb{R}^{2 n}$ of $\widehat{Z}$ such that $0<\operatorname{dist}\left(\widehat{Z}, U_{\varepsilon}\right) \leq \varepsilon$ for all $\varepsilon>0$ sufficiently small we could find a point $P_{\varepsilon} \in \partial\left(N \backslash U_{\varepsilon}\right) \cap\left(\overline{U_{\varepsilon}} \cap N\right) \cap I\left(N \backslash U_{\varepsilon}\right)$. Note that $\left.\left.\partial\left(N \backslash U_{\varepsilon}\right) \cap\left(\overline{U_{\varepsilon}} \cap N\right)\right)=\left[\left(\partial N \backslash U_{\varepsilon}\right) \cup\left(N \cap \partial U_{\varepsilon}\right)\right] \cap\left(\overline{U_{\varepsilon}} \cap N\right)\right)=$ $\left(\partial N \cap \partial U_{\varepsilon}\right) \cup\left(\partial U_{\varepsilon} \cap N\right)$. Let us consider the trajectory $\tau_{\varepsilon}$ passing through $P_{\varepsilon}$. Let $P_{\varepsilon}=\tau_{\varepsilon}\left(t_{\varepsilon}\right)$. This trajectory must reach the set $\partial O_{\delta} \backslash \partial N \cup\left(\partial O_{\delta} \cap \partial N\right)$ for sufficiently large $\left|t-t_{\varepsilon}\right|$. For, suppose that it is not true. Let, for example, for all $t>t_{\varepsilon}$ this trajectory has no common points with this set. It means that it stays in the set $O_{\delta}$ for all $t>t_{\varepsilon}$ as it cannot leave $N$ through $\partial N$. Consequently, either $\tau_{\varepsilon}(t) \rightarrow \widehat{Z}$ as $t \rightarrow \infty$ or the $\omega$-limit set of the trajectory would be an invariant set contained completely in $Z \cap O_{\delta}$. In the first case we arrive at contradiction with the assumption that $\tau_{\varepsilon} \subset I\left(N \backslash U_{\varepsilon}\right) \nsupseteq \widehat{Z}$. In the second case we arrive at 
contradiction with the fact that $I\left(O_{\delta}\right)=\widehat{Z}$. The same arguments may be used to prove that the considered trajectory must reach the set $\partial O_{\delta} \backslash \partial N \cup\left(\partial O_{\delta} \cap \partial N\right)$ for some $t<t_{\varepsilon}$. So, $\tau_{\varepsilon}(t)$ arrives at this set for the first time for $t>t_{\varepsilon}$ at a point $D_{1 \varepsilon}=\tau_{\varepsilon}\left(t_{\varepsilon}+\delta_{1 \varepsilon}\right)$. Similarly it arrives at this set for the first time for $t<t_{\varepsilon}$ at some point $D_{2 \varepsilon}=\tau_{\varepsilon}\left(t_{\varepsilon}-\delta_{2 \varepsilon}\right)$. It is obvious that, due to the continuity of the solutions with respect to initial conditions and the fact that $\widehat{Z}$ is an invariant subset, we must have $\delta_{1 \varepsilon}, \delta_{2 \varepsilon} \rightarrow \infty$ and $\operatorname{dist}\left(P_{\varepsilon}, \widehat{Z}\right) \rightarrow 0$ as $\varepsilon \rightarrow 0$. Let us divide the trajectory $\tau_{\varepsilon}$ into two parts: $\tau_{\varepsilon+}$ joining $D_{2 \varepsilon}$ and $P_{\varepsilon}$ and $\tau_{\varepsilon-}$ joining $D_{1 \varepsilon}$ and $P_{\varepsilon}$. Let $\widetilde{\tau}_{\varepsilon+}=\tau_{\varepsilon+}\left(t+t_{\varepsilon}-\delta_{2 \varepsilon}\right)$. Then $D_{2 \varepsilon}=\widetilde{\tau}_{\varepsilon+}(0)$ and $P_{\varepsilon}=\widetilde{\tau}_{\varepsilon+}\left(\delta_{2 \varepsilon}\right)$. Analogically, if $\widetilde{\tau}_{\varepsilon-}=\tau_{\varepsilon-}\left(t+t_{\varepsilon}+\delta_{1 \varepsilon}\right)$, then $D_{1 \varepsilon}=\widetilde{\tau}_{\varepsilon-}(0)$ and $P_{\varepsilon}=\widetilde{\tau}_{\varepsilon-}\left(-\delta_{1 \varepsilon}\right)$. By passing with $\varepsilon$ to 0 , we infer that in $Z$ there would exist at least one orbit $\left(\tau_{0+}\right)$ tending to a point $P_{0} \in \widehat{Z}$ as $t \rightarrow \infty$ and at least one orbit $\left(\tau_{0-}\right)$ tending to a point $P_{0} \in \widehat{Z}$ as $t \rightarrow-\infty$. This is a contradiction with the assumptions made. The lemma is proved.

Now, let us consider the points in $\left(Z \cap O_{\delta}\right) \backslash \widehat{Z}$. These points must lie on the trajectories that leave $O_{\delta}$ in forward or backward time direction, as $I\left(O_{\delta}\right)=\widehat{Z}$. Suppose, that we can find a point in $\left(Z \cap O_{\delta}\right) \backslash \widehat{Z}$ lying on a trajectory, which does not tend to $\widehat{Z}$ as $t \rightarrow \infty$ or $t \rightarrow-\infty$. If this trajectory leaves the set $O_{\delta}$ in both time directions, then from the proof of the last lemma it follows that it cannot have points in common with $U$, if $U$ is taken sufficiently small. On the other hand, suppose that we can find a point in $\left(Z \cap O_{\delta}\right) \backslash \widehat{Z}$ lying on a trajectory, which stays in $O_{\delta}$ for all $t$ sufficiently large (or all $(-t)$ sufficiently large) and does not tend to $\widehat{Z}$. It follows that the $\omega$-limit ( $\alpha$-limit) set of this trajectory would be an invariant set in $O_{\delta}$ different from $\widehat{Z}$. But this is impossible according to our assumptions. In consequence $I(N \backslash U)=I(N) \backslash\left(\widehat{Z} \cup A^{+} \cup A^{-}\right)$. Thus we may write

$$
\begin{aligned}
& I(N \backslash U) \cap \partial(N \backslash U) \\
& \quad=I(N \backslash U) \cap(\partial N \backslash U) \cup I(N \backslash U) \cap(\partial U \cap N) \\
& \quad=I(N \backslash U) \cap \partial N \backslash I(N \backslash U) \cap U \cup I(N \backslash U) \cap(\partial U \cap N) \\
& \quad=\left[I(N) \backslash \widehat{Z} \backslash A^{+} \backslash A^{-}\right] \cap \partial N \backslash I(N \backslash U) \cap U \cup I(N \backslash U) \cap(\partial U \cap N) .
\end{aligned}
$$

The set $I(N \backslash U) \cap U$ is empty, whereas, the set $I(N \backslash U) \cap(\partial U \cap N)$ is empty according to Lemma 42. In consequence

$$
\begin{aligned}
I(N \backslash U) \cap \partial(N \backslash U) & =\left[I(N) \backslash\left(\widehat{Z} \cup A^{+} \cup A^{-}\right)\right] \cap \partial N \\
& =I(N) \cap \partial N \backslash\left[\widehat{Z} \cup A^{+} \cup\left(A^{-}\right] \cap \partial N\right. \\
& =I(N) \cap \partial N \backslash\left(\widehat{Z} \cup A^{+} \cup A^{-}\right) .
\end{aligned}
$$

That proves Lemma 20. 


\section{REFERENCES}

[1] S. Chow And J. K. Hale, Methods of Bifurcation Theory, Springer-Verlag, 1982.

[2] C. C. Conley, Isolated invariant sets and generalized Morse index, CBMS Regional Conf. Ser. in Math. 38 (1978).

[3] _ A qualitative singular perturbation theorem, Global Theory of Dynamical Systems, Lecture Notes in Math., vol. 819, Springer-Verlag, Berlin, 1981.

[4] C. C. Conley and R. Gardner, An application of the generalized Morse index to travelling wave solutions of a competitive reaction diffusion model, Indiana Univ. Math. J. 33 (1989), 319-343.

[5] E. C. M. Crooks, On the Vol'pert theory of travelling wave solutions for parabolic equations, Nonlinear Anal. 26 (1996), 1621-1642.

[6] E. C. M. Crooks And J. F. Toland, Travelling waves for reaction-diffusion-convection systems, Topol. Methods Nonlinear Anal. 11 (1998), 19-43.

[7] W. Eckhaus, A. van Harten and Z. Peradzyński, A singularly perturbed free boundary problem describing a laser sustained plasma, SIAM J. Appl. Math. 45 (1985), 1-31.

[8] - Plasma produced by a laser in a medium with convection and free surface satisfying a Hamilton-Jacobi equations, Physica D, vol. 27, 1987, pp. 90-112.

[9] P. Fife, Mathematical Aspects of Reacting and Diffusing Systems, Lecture Notes in Math., vol. 28, Springer, New York, 1979.

[10] F. R. Gantmaher, Teoria Matric, Nauka, 1988. (in Russian)

[11] B. KAŹMIERCZAK, Heteroclinic connections in a realistic model of laser sustained plasma, Nonlinear Anal. 29 (1997), 247-264.

[12] B. KAŹMIERCZAK AND Z. PERADZYŃSKi, Heteroclinic solutions for a system of strongly coupled ODEs, Math. Meth. in Appl. Scien. 19 (1996), 451-461.

[13] K. Mischaikov, Conley index theory, Dynamical Systems, Lecture Notes in Math., vol. 1609, Montecatini Terme, 1994.

[14] K. Mischaikov and V. Hutson, Travelling waves for mutualist species, SIAM J. Math. Anal. 24 (1993), 987-1008.

[15] J. D. Murray, Mathematical Biology, Springer, Berlin Heidelberg, 1993.

[16] Z. Peradzý́ski, Continuous optical discharge, properties and modelling, Invited Papers, vol. ICPIG XXI, Bochum, 1993.

[17] J. Smoller, Shock Waves and Reaction Diffusion Systems, Springer-Verlag, 1983.

[18] A. Volpert, V. Volpert and V. Volpert, Travelling Wave Solutions of Parabolic Systems, Amer. Math. Soc., Providence, 1994.

BOGDAN KAŹMIERCZAK

Institute of Fundamental

Technological Research

Świętokrzyska 21

00-049 Warsaw, POLAND

E-mail address: bkazmier@ippt.gov.pl

TMNA : Volume $17-2001-\mathrm{N}^{\circ} 2$ 